\title{
Boundary integral equations for dynamic rupture propagation on vertical complex fault system in half-space: Theory*
}

\author{
Haiming Zhang ${ }^{1, \star} \quad$ Xiaoping Rui ${ }^{2}$ and Wenbo Zhang ${ }^{3}$ \\ ${ }^{1}$ Department of Geophysics, School of Earth and Space Sciences, Peking University, Beijing 100871, China \\ ${ }^{2}$ College of Resources and Environment, Graduate University of Chinese Academy of Sciences, Beijing 100049, China \\ ${ }^{3}$ College of Earth Sciences, Graduate University of Chinese Academy of Sciences, Beijing 100049, China
}

\begin{abstract}
The boundary integral equation method (BIEM) is now widely used in numerical studies on earthquake rupture dynamics, and is proved to be a powerful tool to deal with problems on complex fault system. However, since this method heavily lies on the specific forms of Green's function and only the Green's function in full-space has a closed analytic expression, it is usually limited to a full-space medium. In this study, as a first step to extend this method to an arbitrary complex fault system in half-space, the boundary integral equations (BIEs) for dynamic strike-slip on vertical complex fault system in half-space are derived based on exact Green's function for isotropic and homogeneous half-space. Effect of the geometry of the complex fault system are dealt with carefully. Final BIEs is composed of two parts: contribution from full-space, which has been thoroughly investigated by Aochi and his co-workers by using the Green's function for full-space, and that from free surface, which is studied in detail in this study.
\end{abstract}

Key words: seismic source dynamics; boundary integral equation; complex fault system; free surface CLC number: P315.3 Document code: A

\section{Introduction}

Large devastating earthquakes usually occur along with extensive breaks on surface. Taking the large earthquakes occurred in China in the past decade as an example, we can find the $1999 M_{\mathrm{W}} 7.6 \mathrm{Chi}-\mathrm{Chi}$ (Taiwan) earthquake caused more than $100 \mathrm{~km}$ surface rupture on Chelungpu fault (Shin and Teng, 2001), the $2001 M_{\mathrm{S}} 8.1$ Central Kunlun earthquake produced nearly $400 \mathrm{~km}$ surface rupture zone (Lin et al, 2002), and the 2008 $M_{\mathrm{S}} 8.0$ Wenchuan earthquake caused about $240 \mathrm{~km}$ and $72 \mathrm{~km}$ surface ruptures on Beichuan-Yingxiu fault and Guanxian-Jiangyou fault, respectively (Xu et al, 2008). On such a long distance, the rupture will never propagate on a single fault plane. For example, field investigations show that the Wenchuan earthquake ruptured two NW-dipping imbricate reverse faults along the

\footnotetext{
* Received 19 November 2009; accepted in revised form 7 December 2009; published 10 April 2010.

• Corresponding author. e-mail: zhanghm@pku.edu.cn

(c) The Seismological Society of China and Springer-Verlag Berlin Heidelberg 2010.
}

Longmenshan fault zone at the eastern margin of the Tibetan plateau (Xu et al, 2008). To understand the nature of such complicated seismic source processes, it is necessary to study dynamic rupture process on real fault system under the influence of the ground surface.

In the most popular numerical methods to study dynamic processes of earthquakes, such as the finite difference method (FDM), the finite element method (FEM), and the boundary integral equation method (BIEM), the BIEM is proved to be the most powerful tool to deal with complex fault system (e.g., Tada and Yamashita, 1997; Tada et al, 2000; Aochi and Fukuyama, 2002; Aochi and Madariaga, 2003; Aochi et al, 2000, 2002, 2005). Since in the BIEM, partial differential equations, defined in the domain of a medium, are converted into integral equations defined on the boundaries of the domain, the dimension of the problem will be lowered by one (see, e.g., Tada, 2009). This advantage, together with higher resolution near the fault tip where stress concentration appears, makes the BIEM very flexible and suitable in solving the dynamic rupture 
problems on complex fault system. In recent years, the BIEM has been successfully applied not only to the dynamic problem on parallel faults system (Yamashita and Fukuyama, 1996; Kame and Yamashita, 1997), which has usually been investigated by the FDM (Harris et al, 1991; Harris and Day, 1993, 1999), but also to the dynamic propagation and fault interaction problems on vertically intersecting faults and arbitrarily curved faults (Koller et al, 1992; Bouchon and Streiff, 1997; Kame and Yamashita, 1999; Tada and Yamashita, 1997; Aochi et al, 2000, 2002, 2005; Tada et al, 2000; Aochi and Fukuyama, 2002; Aochi and Madariaga, 2003).

However, the medium models in most studies based on the BIEM have been limited to elastic full-space, because the BIEM depends heavily on the Green's function, and only the Green's function of full-space has a closed analytic form, which is crucial for the simplification of the boundary integral equations (BIEs). Full-space model is a good approximation for cases in which the faults are buried at some depth (Oglesby et al, 2000; Zhang and Chen, 2006b), but it is invalid for a large number of great earthquakes in which the ruptures run directly to the free surface and there exists strong interaction between the fault and the free surface (see, e.g., Nielsen 1998). In view of this, Aochi and his co-workers approximated the effect of free surface (Aochi and Fukuyama, 2002; Aochi and Madariga, 2003) by applying the image method (Quin, 1990) and studied dynamic rupture propagation on vertical strike-slip fault system. However, the image method cannot produce exact solutions. If the symmetry of the problem is broken, this method cannot be applied at all.

On the other hand, Zhang and Chen (2006a) and Chen and Zhang (2006) extended the BIEM (shortened to "EBIEM" for simplicity) to strictly including the effect of free surface in the solution by introducing the exact Green's function for half-space. All the singularities, including hypersingularities, are removed carefully by a two-step separation scheme and an algorithm to accelerate computation of multiple oscillatory integrals is presented. Recently, Zhang and Chen (2009) proved that contribution of direct waves to the Green's function for a half-space expressed in a cylindrical coordinate system is strictly equivalent to the well-known analytic Green's function for full-space (Aki and Richards, 2002). Since all of the hypersingularities in BIEs come from the direct waves and contribution of direct waves can be evaluated analytically, this result is very useful in the simplification of the EBIEM. Although the EBIEM can be used to strictly solve dynamic propagation on arbitrarily complex fault system half-space in principle, it is applied to planar fault with a rectangular shape up to now. To consider both the effects of free surface and fault geometry, it is necessary to further extend the EBIEM to complex fault system. It is not an easy task because of the complexity in mathematics.

As a first step to this goal, the BIEs for a special nonplanar fault system (i.e. a vertical piecewise planar fault) embedded in half-space are strictly derived based on the exact Green's function for half-space in this study. According to Zhang and Chen (2009), the kernels in the BIEs can be separated into two parts: contributions from direct waves, which are exactly equal to those computed based on full-space Green's function (Aochi et al, 2000, 2002; Aochi and Madariaga, 2003), and contributions from free surface, which will be treated carefully in this study.

\section{Model setting and symbol convention}

It is difficult to deal with general problems which are involved with both effects free surface and arbitrary geometry of complex fault system. In view of the complexity in mathematics, we consider a fault system consists of vertical piecewise planar faults for simplicity (Figure 1). Slip is restricted in the horizontal direction, i.e., only strike-slip is permitted. Since we are interested in the influence of free surface on the dynamic ruptures, and it is shown in some studies that the effect of free surface can be neglected for buried faults with depth greater than $1 \mathrm{~km}$ (Oglesby et al, 2000; Zhang and Chen, 2006b). We suppose here that all the subfaults intersect the free surface.

As illustrated in Figure 1, vertical subfaults in grey intersect the free surface $\Pi$ with trace (thick line). Two set of coordinates $O-x_{1} x_{2} x_{3}$ and $O^{\prime}-\xi_{1}^{\prime} \xi_{2}^{\prime} \xi_{3}^{\prime}$ are built up for observation point and source point, and quantities expressed in these two coordinates are denoted without and with apostrophe, respectively. For example, $\Delta u$ and $\Delta u^{\prime}$ represent slip in observation coordinate system and source coordinate system, respectively. Axes $x_{3}$ and $x_{3}^{\prime}$ are perpendicular to $\Pi$, and $x_{1}$ and $x_{1}^{\prime}$ are in the direction of the local strike. Coordinate of observation point and source point are denoted by $x_{i}$ and $\xi_{i}(i=1,2,3)$, respectively. $B$ is the starting point of the trace. The fault system consists of $Q$ subfaults. Starting from point $B$, 


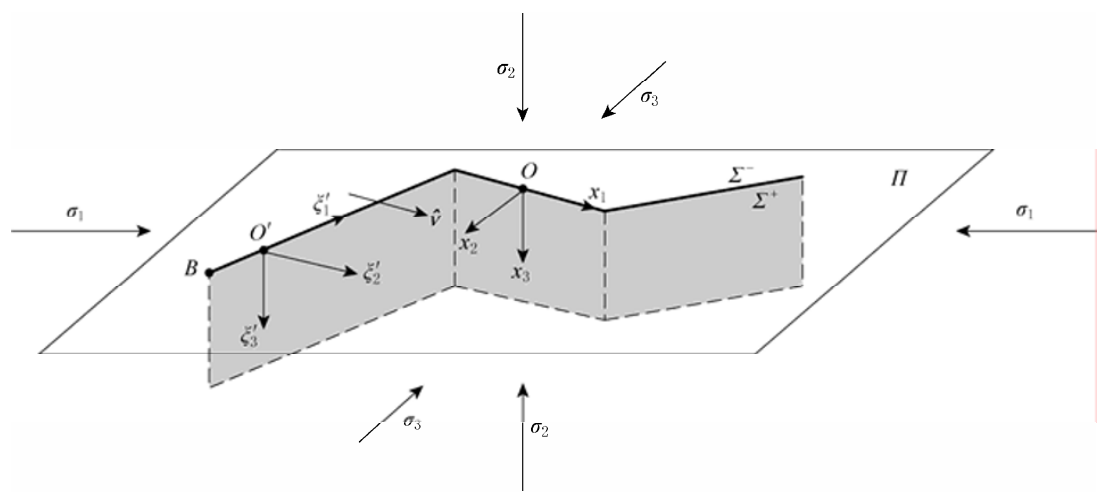

Figure 1 Geometry of the model in this study. Plane $\Pi$ is the free surface, and vertical subfaults are marked in grey. $\sigma_{1}, \sigma_{2}$ and $\sigma_{3}$ are components of principal stress in the source region. Fault is composed of two contact planes $\Sigma^{+}$and $\Sigma^{-}$. $\hat{\boldsymbol{v}}$ is a unit vector pointing from $\Sigma^{-}$to $\Sigma^{+}$. Thick line on $\Pi$ denote the trace of the fault system. $O-x_{1} x_{2} x_{3}$ and $O^{\prime}-\xi_{1}^{\prime} \xi_{2}^{\prime} \xi_{3}^{\prime}$ are coordinates of observation point and source point, respectively. $B$ is the starting point of the trace.

the serial number of the $q$ th subfault is marked as $q(1 \leq$ $q \leq Q)$. Fault is composed of two contact planes $\Sigma^{+}$and $\Sigma^{-}$, and $\hat{\boldsymbol{v}}$ is the unit vector pointing from $\Sigma^{-}$to $\Sigma^{+}$.

The following conventions are used. Subscripts or superscripts in Roman letters $(i, j, k, \cdots)$ run over 1,2 and 3, while those in Greek letters $(\alpha, \beta, \cdots)$ run over 1 and 2. Summation convention is applied. Quantities in frequency domain are represented in $(\widetilde{*})$. Derivatives with respect to $x_{i}$ and $\xi_{i}$ are marked as $\partial_{i}(\cdot)$ and $(\cdot)_{, i}$, respectively. Vectors are written in bold italic letters. Note that in some cases, the same letter will have different meanings. For example, $k$ denotes the serial number of time in discrete BIEs, while it also denotes horizontal wavenumber in Green's function somewhere else. However, the meaning can be inferred from context without confusion.

\section{General boundary integral equations}

In this section, based on the representation theorem in general form, the general traction BIEs, which is independent of the specific forms of the Green's functions, are established.

Starting from the representation theorem expressed in observation coordinate system (e.g., Aki and Richards, 2002), we obtain

$$
\begin{gathered}
\tilde{u}_{n}(\boldsymbol{x}, \omega)= \\
\sum_{q=1}^{Q} \iint_{\Sigma_{q}} \Delta \widetilde{u}_{i}(\xi, \omega) c_{i j k l} v_{j}(\xi) \widetilde{G}_{n k, l}(\boldsymbol{x}, \xi, \omega) \mathrm{d} S(\xi)
\end{gathered}
$$

where $u_{n}$ on the left hand side is the displacement at $\boldsymbol{x}$,
$\Delta u_{i}$ is the slip on the $q$ th subfault, $c_{i j k l}$ is the elastic coefficient (for isotropic medium, there are only two non-zero components $\lambda$ and $\mu$ ), $G_{n k}$ is the Green's function, and $\omega$ is the circular frequency. In order to build up the BIEs, $\boldsymbol{x}$ must be put on the fault plane. However, equation (1) is valid only for inner point. Zhang and Chen (2006a) dealt with this difficulty carefully, they transformed the point on the fault plane to an inner point by excavating a small neighborhood of the point and taking limit. This is the origin of hypersingularity in the BIEs. Detailed analysis revealed that the singularity in the BIEs arises only from direct waves, while free surface does not cause any singularity (Zhang, 2004). This implies that if the whole BIEs can be divided into two parts: one is related to direct waves, and the other is related to free surface, we only need to deal with the latter. The former is exactly the BIEs based on full-space Green's function (Aochi et al, 2000, 2002). Fortunately, this is true when the half-space Green's function is expressed as wave-number integrals in frequency domain, as shown by Zhang and Chen (2009). Therefore, in the following, we will focus on the part of free surface, and put the observation point $x$ directly on the fault (denoted as $\boldsymbol{x}^{P}$ ).

Note that when the observation point $\boldsymbol{x}^{P}$ and the source point $\xi$ are located on the same subfault, normal vector $\hat{\boldsymbol{v}}$ is in the same direction as axis $x_{2}$, the derivation will be greatly simplified. According to this, equation (1) can be rewritten as

$$
\widetilde{u}_{n}\left(\left.\boldsymbol{x}^{P}\right|_{\Sigma_{q_{0}}}, \omega\right)=\widetilde{u}_{n}^{(\mathrm{nc})}\left(\left.\boldsymbol{x}^{P}\right|_{\Sigma_{q_{0}}}, \omega\right)+\widetilde{u}_{n}^{(\mathrm{c})}\left(\left.\boldsymbol{x}^{P}\right|_{\Sigma_{q_{0}}}, \omega\right),
$$


where

$$
\begin{gathered}
\tilde{u}_{n}^{(\mathrm{nc})}\left(\left.\boldsymbol{x}^{\mathrm{P}}\right|_{\Sigma_{q_{0}}}, \omega\right)= \\
\sum_{q=1 \wedge q \neq q_{0}}^{Q} \iint_{\Sigma_{q}} \Delta \widetilde{u}_{i}(\xi, \omega) c_{i j k l} \nu_{j}(\xi) \widetilde{G}_{n k, l}\left(\left.\boldsymbol{x}^{\mathrm{P}}\right|_{\Sigma_{q_{0}}}, \boldsymbol{\xi}, \omega\right) \mathrm{d} S(\boldsymbol{\xi})
\end{gathered}
$$

and

$$
\begin{gathered}
\tilde{u}_{n}^{(\mathrm{c})}\left(\left.\boldsymbol{x}^{\mathrm{P}}\right|_{\Sigma_{q_{0}}}, \omega\right)= \\
\iint_{\Sigma_{q_{0}}} \Delta \widetilde{u}_{i}(\xi, \omega) c_{i j k l} \nu_{j}(\xi) \widetilde{G}_{n k, l}\left(\left.\boldsymbol{x}^{\mathrm{P}}\right|_{\Sigma_{q_{0}}}, \xi, \omega\right) \mathrm{d} S(\xi)
\end{gathered}
$$

are non-coplanar part (denoted by superscript "(nc)") and coplanar part (denoted by superscript "(c)") of the displacement $\tilde{u}_{n}$, respectively. $\left.\boldsymbol{x}^{P}\right|_{\Sigma_{q 0}}$ is the observation point $\boldsymbol{x}^{P}$ located on subfault $\Sigma_{q_{0}}$. We will deal with these two cases in the following two subsections.

\subsection{Non-coplanar part of the general BIEs}

In this case, $\hat{\boldsymbol{v}}$ is not in the direction of axis $x_{2}$, as shown in Figure 2. Both $\Delta \widetilde{u}_{1}$ and $\Delta \widetilde{u}_{2}$ are not zero in the observation coordinate system, whose origin $O$ is located on observation point $\boldsymbol{x}^{\mathrm{P}}$. The angle measured counter clockwise from subfault $\Sigma_{q_{0}}$ to subfault $\Sigma_{q}$ is marked as $\theta_{q, q_{0}}$. Then,

$$
\begin{aligned}
& \hat{\boldsymbol{v}}=\left(v_{1}, v_{2}, v_{3}\right)=\left(\sin \left(\theta_{q, q_{0}}\right), \cos \left(\theta_{q, q_{0}}\right), 0\right) \text {. } \\
& \widetilde{\tau}_{21}^{(\text {nc) }}=\mu\left(\partial_{2} \tilde{u}_{1}^{(\mathrm{nc})}+\partial_{1} \tilde{u}_{2}^{(\mathrm{nc})}\right)=\mu^{2} \sum_{q=1 \wedge q \neq q 0}^{Q} \iint_{\Sigma_{q}^{\prime}}\left\{p\left(\eta \Delta \widetilde{u}_{1}+\Delta \widetilde{u}_{2}\right)\left(\partial_{1} \widetilde{G}_{2 k, k}+\partial_{2} \widetilde{G}_{1 k, k}\right)+2 \eta \Delta \widetilde{u}_{1}\left(\partial_{1} \widetilde{G}_{21,1}+\partial_{2} \widetilde{G}_{11,1}\right)+\right. \\
& \left.2 \Delta \widetilde{u}_{2}\left(\partial_{1} \widetilde{G}_{22,2}+\partial_{2} \widetilde{G}_{12,2}\right)+\left(\Delta \widetilde{u}_{1}+\eta \Delta \widetilde{u}_{2}\right)\left(\partial_{1} \widetilde{G}_{21,2}+\partial_{1} \widetilde{G}_{22,1}+\partial_{2} \widetilde{G}_{11,2}+\partial_{2} \widetilde{G}_{12,1}\right)\right\}_{\xi_{2}=L_{q}\left(\xi_{1}\right)} \mathrm{d} \xi_{1} \mathrm{~d} \xi_{3}, \\
& \widetilde{\tau}_{22}^{(\mathrm{nc})}=\lambda \partial_{k} \widetilde{u}_{k}^{(\text {nc) }}+2 \mu \partial_{2} \widetilde{u}_{2}^{(\mathrm{nc})}=\mu^{2} \sum_{q=1 \wedge q \neq q_{0}}^{Q} \iint_{\Sigma_{q}^{\prime}}\left\{p\left(\eta \Delta \widetilde{u}_{1}+\Delta \widetilde{u}_{2}\right)\left[p \partial_{1} \widetilde{G}_{1 k, k}+(p+2) \partial_{2} \widetilde{G}_{2 k, k}+p \partial_{3} \widetilde{G}_{3 k, k}\right]+\right. \\
& 2 \eta \Delta \widetilde{u}_{1}\left[p \partial_{1} \widetilde{G}_{11,1}+(p+2) \partial_{2} \widetilde{G}_{21,1}+p \partial_{3} \widetilde{G}_{31,1}\right]+2 \Delta \widetilde{u}_{2}\left[p \partial_{1} \widetilde{G}_{12,2}+(p+2) \partial_{2} \widetilde{G}_{22,2}+p \partial_{3} \widetilde{G}_{32,2}\right]+ \\
& \left.\left(\Delta \widetilde{u}_{1}+\eta \Delta \widetilde{u}_{2}\right)\left[p\left(\partial_{1} \widetilde{G}_{11,2}+\partial_{1} \widetilde{G}_{12,1}\right)+(p+2)\left(\partial_{2} \widetilde{G}_{21,2}+\partial_{2} \widetilde{G}_{22,2}\right)+p\left(\partial_{3} \widetilde{G}_{31,2}+\partial_{3} \widetilde{G}_{32,1}\right)\right]\right\}_{\xi_{2}=L_{q}\left(\xi_{1}\right)} \mathrm{d} \xi_{1} \mathrm{~d} \xi_{3},
\end{aligned}
$$

where, $\Sigma_{q}^{\prime}$ is the projection of $\Sigma_{q}$ on $\Sigma_{q_{0}}$, and $\xi_{2}=L_{q}\left(\xi_{1}\right)$ is the equation of $\xi_{2}$ expressed in $\xi_{1}$, which is obtained from the equation of $\Sigma_{q}$ in the observation coordinate system. $p=\lambda / \mu$ and $\eta=v_{1} / v_{2}$.

Notice that

$$
\frac{\partial}{\partial \xi_{3}} \widetilde{G}_{i j}=\frac{\mathrm{d}}{\mathrm{d} \xi_{3}} \widetilde{G}_{i j}, \quad \frac{\partial}{\partial \xi_{1}} \widetilde{G}_{i j}=\frac{\mathrm{d}}{\mathrm{d} \xi_{1}} \widetilde{G}_{i j}+\eta \frac{\partial}{\partial \xi_{2}} \widetilde{G}_{i j},
$$

We can obtain

$$
\iint_{\Sigma_{q}^{\prime}} \Delta \tilde{u}_{\alpha} \widetilde{G}_{i j, 3} \mathrm{~d} \xi_{1} \mathrm{~d} \xi_{3}=-\iint_{\Sigma_{q}^{\prime}} \Delta \widetilde{u}_{\alpha, 3} \widetilde{G}_{i j} \mathrm{~d} \xi_{1} \mathrm{~d} \xi_{3},
$$

$$
\begin{gathered}
\iint_{\Sigma_{q}^{\prime}} \Delta \widetilde{u}_{\alpha} \widetilde{G}_{i j, 1} \mathrm{~d} \xi_{1} \mathrm{~d} \xi_{3}= \\
-\iint_{\Sigma_{q}^{\prime}}\left(\Delta \widetilde{u}_{\alpha, 1}-\eta \Delta \widetilde{u}_{\alpha, 2}\right) \widetilde{G}_{i j} \mathrm{~d} \xi_{1} \mathrm{~d} \xi_{3}+ \\
\eta \iint_{\Sigma_{q}^{\prime}} \Delta \widetilde{u}_{\alpha} \widetilde{G}_{i j, 2} \mathrm{~d} \xi_{1} \mathrm{~d} \xi_{3} .
\end{gathered}
$$

Applying the relations in equation (7) and $\partial_{\alpha} \widetilde{G}_{i j}=-\widetilde{G}_{i j, \alpha}$ produces the regularized integrals (listed in equation (A1) in Appendix A). Substituting equation (A1) into equation (6), we finally obtain 


$$
\begin{aligned}
\widetilde{\tau}_{21}^{(\mathrm{nc})}= & p \kappa_{\alpha}^{(1)}\left(U_{\alpha 211}^{\mathrm{I}}+\eta U_{\alpha 212}^{\mathrm{I}}-\eta^{2} W_{\alpha 21}^{\mathrm{I}}+U_{\alpha 222}^{\mathrm{I}}-\eta W_{\alpha 22}^{\mathrm{I}}+U_{\alpha 231}^{\mathrm{II}}+U_{\alpha 112}^{\mathrm{II}}-\eta W_{\alpha 11}^{\mathrm{I}}-W_{\alpha 12}^{\mathrm{II}}+U_{\alpha 132}^{\mathrm{II}}\right)+ \\
& \kappa_{\alpha}^{(2)}\left(U_{\alpha 212}^{\mathrm{I}}-\eta W_{\alpha 21}^{\mathrm{I}}+U_{\alpha 221}^{\mathrm{I}}+\eta U_{\alpha 222}^{\mathrm{I}}-\eta^{2} W_{\alpha 22}^{\mathrm{I}}-W_{\alpha 11}^{\mathrm{I}}+U_{\alpha 122}^{\mathrm{I}}-\eta W_{\alpha 12}^{\mathrm{I}}\right)+ \\
& 2 \eta\left(U_{1211}^{\mathrm{I}}+\eta U_{1212}^{\mathrm{I}}-\eta^{2} W_{121}^{\mathrm{I}}+U_{1112}^{\mathrm{I}}-\eta W_{111}^{\mathrm{I}}\right)+2\left(U_{2222}^{\mathrm{I}}-\eta W_{222}^{\mathrm{I}}-W_{212}^{\mathrm{I}}\right), \\
\widetilde{\tau}_{22}^{(\mathrm{nc})}= & p \kappa_{\alpha}^{(1)}\left[p\left(U_{\alpha 111}^{\mathrm{I}}+\eta U_{\alpha 112}^{\mathrm{I}}-\eta^{2} W_{\alpha 11}^{\mathrm{I}}+U_{\alpha 122}^{\mathrm{I}}-\eta W_{\alpha 12}^{\mathrm{I}}+U_{\alpha 131}^{\mathrm{II}}\right)+(p+2)\left(U_{\alpha 212}^{\mathrm{I}}-\eta W_{\alpha 21}^{\mathrm{I}}-W_{\alpha 22}^{\mathrm{I}}+U_{\alpha 232}^{\mathrm{II}}\right)-\right. \\
& \left.p\left(V_{\alpha 31}^{\mathrm{I}}-\eta W_{\alpha 31}^{\mathrm{II}}-W_{\alpha 32}^{\mathrm{II}}+V_{\alpha 33}^{\mathrm{II}}\right)\right]+\kappa_{\alpha}^{(2)}\left[p\left(U_{\alpha 112}^{\mathrm{I}}-\eta W_{\alpha 11}^{\mathrm{I}}+U_{\alpha 121}^{\mathrm{I}}+\eta U_{\alpha 122}^{\mathrm{I}}-\eta^{2} W_{\alpha 12}^{\mathrm{I}}\right)-\right. \\
& \left.(p+2)\left(W_{\alpha 21}^{\mathrm{I}}-U_{\alpha 221}^{\mathrm{I}}+\eta W_{\alpha 22}^{\mathrm{I}}\right)+P\left(W_{\alpha 31}^{\mathrm{II}}-V_{\alpha 32}^{\mathrm{I}}+\eta W_{\alpha 32}^{\mathrm{II}}\right)\right]+2 \eta\left[p\left(U_{1111}^{\mathrm{I}}+\eta U_{1112}^{\mathrm{I}}-\eta^{2} W_{111}^{\mathrm{I}}\right)+\right. \\
& \left.(p+2)\left(U_{1212}^{\mathrm{I}}-\eta W_{121}^{\mathrm{I}}\right)-p\left(V_{131}^{\mathrm{I}}-\eta W_{131}^{\mathrm{II}}\right)\right]+2\left[p\left(U_{2122}^{\mathrm{I}}-\eta W_{212}^{\mathrm{I}}\right)-(p+2) W_{222}^{\mathrm{I}}+p W_{232}^{\mathrm{II}}\right] .
\end{aligned}
$$

where

$$
\kappa_{\alpha}^{(1)}=\left\{\begin{array}{ll}
\eta, & \text { as } \alpha=1, \\
1, & \text { as } \alpha=2,
\end{array} \quad \kappa_{\alpha}^{(2)}= \begin{cases}1, & \text { as } \alpha=1 \\
\eta, & \text { as } \alpha=2\end{cases}\right.
$$

and expressions for $U_{o i j k}^{\beta}, V_{\alpha j k}^{\beta}$ and $W_{o j i k}^{\beta}$ are listed in equation (B1) in Appendix B.

\subsection{Coplanar part of the general BIEs}

In this case, normal vector $\hat{\boldsymbol{v}}$ is in the same direction of axis $x_{2}$, as shown in Figure 3. In the observation coordinate system, whose origin $O$ is located on observation point $\boldsymbol{x}^{P}, \Delta \widetilde{u}_{2}=0$, and $\hat{\boldsymbol{v}}=\left(v_{1}, v_{2}, v_{3}\right)=(0,1,0)$. Therefore, the coplanar part of the displacement can be expressed simply as

$$
\widetilde{u}_{n}^{(\mathrm{c})}=\mu \iint_{\Sigma_{q_{0}}} \Delta \widetilde{u}_{1}\left(v_{2} \widetilde{G}_{n 1,2}+v_{2} \widetilde{G}_{n 2,1}\right) \mathrm{d}(\xi) .
$$

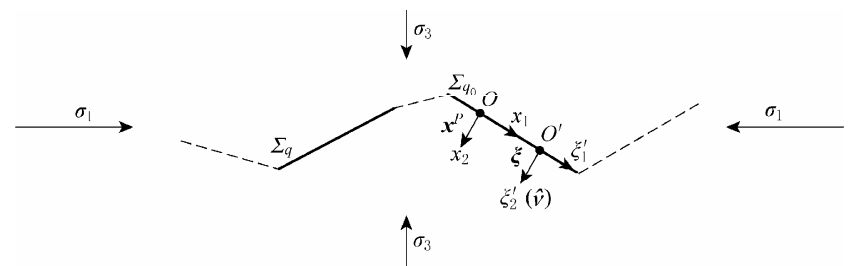

Figure 3 Top view of the fault system for solving coplanar part of solution. Symbols are the same as those in Figure 2. Note that in this case, the source point $\xi$ and observation point $\boldsymbol{x}^{P}$ are located on the same subfault $\Sigma_{q_{0}}$.

Based on equation (10), the shear stress $\widetilde{\tau}_{21}$ and normal stress $\widetilde{\tau}_{22}$ on subfault where the observation point $\boldsymbol{x}^{P}$ is located can be expressed as

$$
\begin{gathered}
\widetilde{\tau}_{21}^{(\mathrm{c})}=\mu\left(\partial_{2} \widetilde{u}_{1}^{(\mathrm{nc})}+\partial_{1} \widetilde{u}_{2}^{(\mathrm{nc})}\right)=\mu^{2} \iint_{\Sigma_{q_{0}}}\left\{\Delta \widetilde { u } _ { 1 } \left(\partial_{2} \widetilde{G}_{12,1}+\right.\right. \\
\left.\left.\partial_{2} \widetilde{G}_{11,2}+\partial_{1} \widetilde{G}_{22,1}+\partial_{1} \widetilde{G}_{21,2}\right)\right\}_{\xi_{2}=0} \mathrm{~d} \xi_{1} \mathrm{~d} \xi_{3}, \\
\widetilde{\tau}_{22}^{(\mathrm{c})}=\lambda \partial_{k} \widetilde{u}_{k}^{(\mathrm{nc})}+2 \mu \partial_{2} \widetilde{u}_{2}^{(\mathrm{nc})}= \\
\mu^{2} \iint_{\Sigma_{q_{0}}}\left\{\Delta \widetilde { u } _ { 1 } \left[(p+2)\left(\partial_{2} \widetilde{G}_{21,2}+\partial_{2} \widetilde{G}_{22,1}\right)+\right.\right. \\
\left.\left.2\left(\partial_{1} \widetilde{G}_{11,2}+\partial_{1} \widetilde{G}_{12,1}+\partial_{3} \widetilde{G}_{31,2}+\partial_{3} \widetilde{G}_{32,1}\right)\right]\right\}_{\xi_{2}=0} \mathrm{~d} \xi_{1} \mathrm{~d} \xi_{3} .
\end{gathered}
$$

In coplanar case, the regularized integrals can be simplified as that in equation (A2) in Appendix A. Combining equation (A2) with equation (11), and noticing that $\widetilde{G}_{21}=\widetilde{G}_{12}$ (Zhang, 2004), we can finally obtain

$$
\begin{gathered}
\widetilde{\tau}_{21}^{(\mathrm{c})}=2 X_{122}+X_{211}-Z_{11}^{\mathrm{I}}, \\
\widetilde{\tau}_{22}^{(\mathrm{c})}=p X_{222}+X_{112}+X_{121}-Y_{32}-p Z_{21}^{\mathrm{I}}+Z_{31}^{\mathrm{II}} .
\end{gathered}
$$

Expressions for $X_{i j k}, Y_{i j k}$ and $Z_{i j k}$ are listed in equation (B2) in Appendix B.

\section{Discrete boundary integral equations}

In this section, we apply the box-like discretization scheme (see, Fukuyama and Madariaga, 1998; Aochi et al, 2000) to obtain the discrete BIEs which are convenient for numerical computation. In the source coordinate system, slip rate can be expressed as

$$
\begin{aligned}
\Delta \dot{u}_{\alpha}^{\prime}\left(\xi^{\prime}, \tau\right)= & \sum_{l, m, n} V_{\alpha}^{\prime l m n}\left[H\left(\xi_{1}^{\prime}-\xi_{1}^{\prime l}\right)-H\left(\xi_{1}^{\prime}-\xi_{1}^{\prime l+1}\right)\right] . \\
& {\left[H\left(\xi_{3}^{\prime}-\xi_{3}^{\prime m}\right)-H\left(\xi_{3}^{\prime}-\xi_{3}^{\prime m+1}\right)\right] } \\
& {\left[H\left(\tau-\tau^{n}+\Delta t\right)-H\left(\tau-\tau^{n}\right)\right] }
\end{aligned}
$$

where $H(\cdot)$ is the Heaviside step function, $\xi_{1}^{\prime l}$ and $\xi_{3}^{\prime m}$ are the coordinates of point who starts from point $B$ and goes $l \Delta s$ along fault system on surface and $m \Delta s$ vertically downward. $\tau^{n}=n \Delta t$, where $\Delta s$ and $\Delta t$ are step size of space and time, respectively. $V^{\prime m n}$ are slip rate measured in source coordinate system. In the following two subsections, we will derive the non-coplanar part and coplanar part of the discrete BIEs, respectively.

\subsection{Non-coplanar part of the discrete BIEs}

Equation (13) is expressed in the source coordinate system. Since the BIEs in equations (8) and (12) are expressed in observation coordinate system, it is convenient to rewrite equation (13) in observation coordinate system as

$$
\Delta \widetilde{u}_{1}(\xi, \omega)=v_{2} \sum_{l, m, n} V_{1}^{\prime l m n} K^{l}\left(\xi_{1}, \xi_{2}\right) L^{m}\left(\xi_{3}\right) \widetilde{S}(\omega),
$$




$$
\Delta \widetilde{u}_{2}(\xi, \omega)=-v_{1} \sum_{l, m, n} V_{1}^{\prime l m n} K^{l}\left(\xi_{1}, \xi_{2}\right) L^{m}\left(\xi_{3}\right) \widetilde{S}(\omega),
$$

where

$$
\begin{gathered}
K^{l}\left(\xi_{1}, \xi_{2}\right)=H\left(v_{2} \xi_{1}-v_{1} \xi_{2}+\left(\xi_{1}^{\prime}\right)_{O}-\xi_{1}^{\prime l}\right)- \\
H\left(v_{2} \xi_{1}-v_{1} \xi_{2}+\left(\xi_{1}^{\prime}\right)_{O}-\xi_{1}^{\prime l+1}\right), \\
L^{m}\left(\xi_{3}\right)=H\left(\xi_{3}-\xi_{3}^{\prime m}\right)-H\left(\xi_{3}-\xi_{3}^{\prime m+1}\right), \\
\widetilde{S}^{n}(\omega)=\frac{1}{\omega^{2}}\left(1-\mathrm{e}^{\mathrm{i} \omega \Delta t}\right) \mathrm{e}^{-\mathrm{i} \omega n \Delta t} .
\end{gathered}
$$

with $\left(\xi_{1}^{\prime}\right)_{O}$ and $\left(\xi_{2}^{\prime}\right)_{O}$ the values of the origin $O$ of the observation coordinate system (i.e. the observation point) in the axes $x_{1}^{\prime}$ and $x_{2}^{\prime}$ of source coordinate system, respectively.

From equation (14), it is easy to write

$$
\begin{gathered}
{\left.\left[\Delta \widetilde{u}_{1,1}(\xi, \omega)-\eta \Delta \widetilde{u}_{1,2}(\xi, \omega)\right]\right|_{\xi_{2}=L_{q}\left(\xi_{1}\right)}=} \\
v_{2} \sum_{l, m, n} V_{1}^{\prime l m n} M^{l}\left(\xi_{1}\right) L^{m}\left(\xi_{3}\right) \widetilde{S}^{n}(\omega), \\
{\left.\left[\Delta \widetilde{u}_{2,1}(\xi, \omega)-\eta \Delta \widetilde{u}_{2,2}(\xi, \omega)\right]\right|_{\xi_{2}=L_{q}\left(\xi_{1}\right)}=} \\
-v_{1} \sum_{l, m, n} V_{1}^{\prime l m n} M^{l}\left(\xi_{1}\right) L^{m}\left(\xi_{3}\right) \widetilde{S}^{n}(\omega),
\end{gathered}
$$

$$
\begin{gathered}
\left.\Delta \widetilde{u}_{1,3}(\xi, \omega)\right|_{\xi_{2}=L_{q}\left(\xi_{1}\right)}= \\
v_{2} \sum_{l, m, n} V_{1}^{\prime l m n} N^{l}\left(\xi_{1}\right) O^{m}\left(\xi_{3}\right) \widetilde{S}^{n}(\omega), \\
\left.\Delta \widetilde{u}_{2,3}(\xi, \omega)\right|_{\xi_{2}=L_{q}\left(\xi_{1}\right)}= \\
-v_{1} \sum_{l, m, n} V_{1}^{\prime l m n} N^{l}\left(\xi_{1}\right) O^{m}\left(\xi_{3}\right) \widetilde{S}^{n}(\omega),
\end{gathered}
$$

where

$$
\begin{gathered}
M^{l}\left(\xi_{1}\right)=\delta\left(\xi_{1}+v_{1}\left(\xi_{1}^{\prime}\right)_{O}+v_{2}\left(\xi_{1}^{\prime}\right)_{O}-v_{2} \xi_{1}^{\prime l}\right)- \\
\delta\left(\xi_{1}+v_{1}\left(\xi_{2}^{\prime}\right)_{O}+v_{2}\left(\xi_{1}^{\prime}\right)_{O}-v_{2} \xi_{1}^{\prime+1}\right), \\
N^{l}\left(\xi_{1}\right)=H\left(\xi_{1}+v_{1}\left(\xi_{1}^{\prime}\right)_{O}+v_{2}\left(\xi_{1}^{\prime}\right)_{O}-v_{2} \xi_{1}^{\prime l}\right)- \\
H\left(\xi_{1}+v_{1}\left(\xi_{2}^{\prime}\right)_{O}+v_{2}\left(\xi_{1}^{\prime}\right)_{O}-v_{2} \xi_{1}^{\prime l+1}\right), \\
O^{m}\left(\xi_{3}\right)=\delta\left(\xi_{3}-\xi_{3}^{\prime m}\right)-\delta\left(\xi_{3}-\xi_{3}^{\prime m+1}\right) .
\end{gathered}
$$

with $\delta(\cdot)$ the Dirac $\delta$ function.

Substituting equations (14) and (16) into equations (8) and (B1) in Appendix B and discretizing the observation coordinates, we finally obtain the non-coplanar part of the discrete BIEs as

$$
\tau_{2 \alpha}^{(\mathrm{nc}) i j k}=\sum_{\substack{l, m, n \\(n \leq k)}} C_{\alpha}^{(\mathrm{nc}) i j k ; m n} V_{1}^{\prime l m n},
$$

where

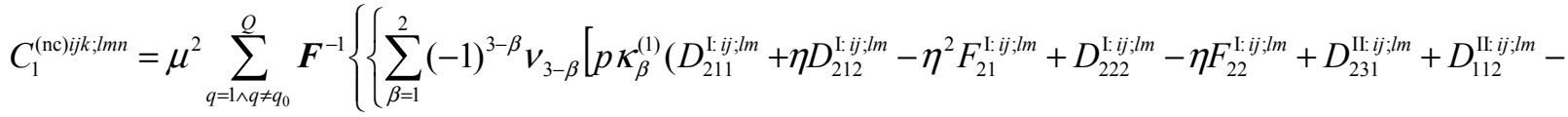

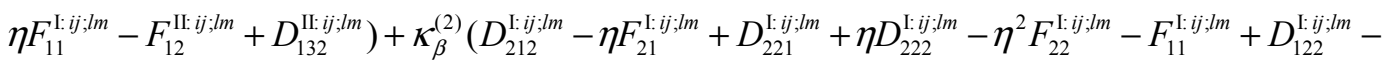

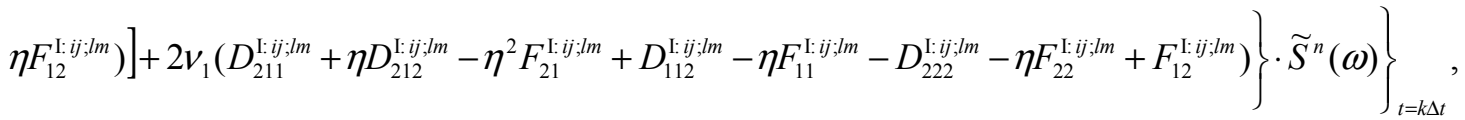

$$
\begin{aligned}
& C_{2}^{(\mathrm{nc}) i j k ; l m n}=\mu^{2} \sum_{q=1 \wedge q \neq q_{0}}^{Q} \boldsymbol{F}^{-1}\left\{\sum _ { \beta = 1 } ^ { 2 } ( - 1 ) ^ { 3 - \beta } v _ { 3 - \beta } \left[p \boldsymbol { \kappa } _ { \beta } ^ { ( 1 ) } \left(p \left(D_{111}^{\mathrm{I} i j ; l l m}+\eta D_{112}^{\mathrm{I}: i j / l m}-\eta^{2} F_{11}^{\mathrm{I}: i j ; l m}+D_{122}^{\mathrm{I}: i j ; l m}-\eta F_{12}^{\mathrm{I}: i j ; l m}+\right.\right.\right.\right.
\end{aligned}
$$

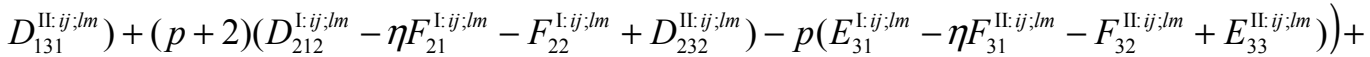

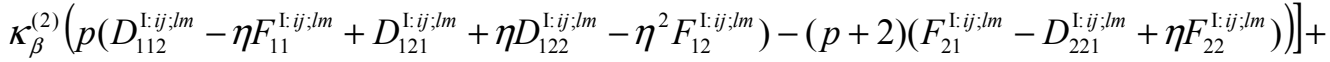

$$
\begin{aligned}
& p\left(F_{31}^{\mathrm{I}: i j: l m}-\varepsilon_{32}^{\mathrm{I}: i j: l m}+\eta F_{32}^{\mathrm{II}: i j: l m}\right)+
\end{aligned}
$$

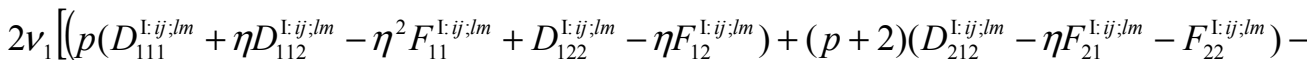

$$
\begin{aligned}
& \left.\left.\left.\left.p\left(E_{31}^{\mathrm{I}: i j: l m}-\eta F_{31}^{\mathrm{II} i j: l m}-F_{32}^{\mathrm{I}: i j: l m}\right)\right)\right]\right\} \cdot \widetilde{S}^{n}(\omega)\right\}_{t=k \Delta t},
\end{aligned}
$$

$\boldsymbol{F}^{-1}$ is the operator of inverse Fourier transform, and expressions for $D_{r s t}^{\beta: i j: l m}, E_{r s}^{\beta: i j ; l m}$ and $F_{r s}^{\beta: i j: l m}$ are listed in equation (C1) in Appendix C.

\subsection{Coplanar part of the discrete BIEs}

In this case, slip functijon in observation coordinate

$$
\begin{gathered}
\Delta \widetilde{u}_{1}(\xi, \omega)=\sum_{l, m, n} V_{1}^{\prime l m n}\left[H\left(\xi_{1}+\left(\xi_{1}^{\prime}\right)_{O}-\xi_{1}^{\prime l}\right)-\right. \\
\left.H\left(\xi_{1}+\left(\xi_{1}^{\prime}\right)_{O}-\xi_{1}^{\prime l+1}\right)\right] \cdot\left[H\left(\xi_{3}-\xi_{3}^{\prime m}\right)-\right. \\
\left.H\left(\xi_{3}-\xi_{3}^{\prime m+1}\right)\right] \widetilde{S}^{n}(\omega),
\end{gathered}
$$
system can be accordingly expressed as 
therefore

$$
\begin{gathered}
\Delta \tilde{u}_{1,1}(\xi, \omega)=\sum_{l, m, n} V_{1}^{\prime l m n}\left[\delta\left(\xi_{1}+\left(\xi_{1}^{\prime}\right)_{O}-\xi_{1}^{\prime l}\right)-\right. \\
\left.\delta\left(\xi_{1}+\left(\xi_{1}^{\prime}\right)_{O}-\xi_{1}^{\prime l+1}\right)\right] \cdot\left[H\left(\xi_{3}-\xi_{3}^{\prime m}\right)-\right. \\
\left.H\left(\xi_{3}-\xi_{3}^{\prime m+1}\right)\right] \tilde{S}^{n}(\omega) .
\end{gathered}
$$

Substituting equation (21) into equation (B2) in Appendix B and discretizing the observation coordinates produces a similar coplanar part of the discrete BIEs as that for non-coplanar part

$$
\tau_{2 \alpha}^{(\mathrm{c}) i j k}=\sum_{\substack{l, m, n \\(n \leq k)}} C_{\alpha}^{(\mathrm{c}) i j k ; l m n} V_{1}^{\prime l m n}
$$

where

$$
\begin{gathered}
C_{1}^{(\mathrm{nc}) i j k ; l m n}=\mu^{2} \boldsymbol{F}^{-1}\left\{\left(2 I_{122}^{i j ; l m}+I_{221}^{i j: l m}-\right.\right. \\
\left.\left.K_{11}^{\mathrm{I} i j ; l m}\right) \cdot \widetilde{S}^{n}(\omega)\right\}_{t=k \Delta t}, \\
C_{2}^{(\mathrm{nc}) i j k ; l m n}=\mu^{2} \boldsymbol{F}^{-1}\left\{\left(p I_{222}^{i j ; l m}+I_{112}^{i j ; l m}+I_{121}^{i j ; l m}-\right.\right. \\
\left.\left.J_{32}^{i j: l m}-p K_{12}^{\mathrm{I}: i j ; l m}+K_{31}^{\mathrm{II}: i j ; l m}\right) \cdot \widetilde{S}^{n}(\omega)\right\}_{t=k \Delta t} \cdot
\end{gathered}
$$

Expressions for $I_{r s t}^{i j: l m}, J_{r s}^{i j / l m}$ and $K_{r s}^{\beta: i j: l m}$ are listed in equation (C2) in Appendix C.

So far, we have obtained the non-coplanar part and coplanar part of the discrete BIEs, as in equations (18) and (22). Note that these equations are contributions of free surface. The contributions for direct waves are exactly the same as those for full-space, which can be evaluated by conventional BIEs based on full-space Green's function, such as those in Aochi et al (2000). In view of this, the complete BIEs for solving dynamic rupture propagation on vertical complex fault system in half-space can be expressed as $\tau_{2 \alpha}^{i j k}=\tau_{2 \alpha}^{i j 0}+\sum_{\substack{l, m, n \\(n \leq k)}}\left(P_{\alpha}^{i j k ; l m n}+C_{\alpha}^{(\mathrm{nc}) i j k ; l m n}+C_{\alpha}^{(\mathrm{c}) i j k ; l m n}\right) V_{1}^{\prime l m n}$,

where $\tau_{2 \alpha}^{i j k}$ on the left hand side is the absolute shear stress, $\tau_{2 \alpha}^{i j 0}$ on the right hand side is the initial stress, $P_{\alpha}^{i j k ; m n}$ is the integral kernel for full-space, and detailed expressions should be referred to Aochi et al (2000).

\section{Green's function for half-space and its partial derivatives}

According to the discrete BIEs equation (24), it is crucial to compute the kernels full-space kernel $P_{\alpha}^{i j k: l m n}$ and kernels of free surface $C_{\alpha}^{(\mathrm{nc}) i j k ; m n}$ and $C_{\alpha}^{(\mathrm{c}) i j k ; l m n}$, which are expressed in integrals of partial derivatives of Green's function with respect to space coordinate, as shown in equations (19), (23), (C1) and (C2) in Appendix C. Table 1 lists all the components of partial derivatives of Green's function to be computed.

Table 1 Partial derivatives of Green's function to be computed

\begin{tabular}{cc}
\hline Derivative of Green's function & Components to be computed: $(r s, t)$ or $(r s)$ \\
\hline$\widetilde{G}_{r s, t}$ & $(11,1)(11,2)(12,1)(12,2)(13,1)$ \\
$\partial_{3} \widetilde{G}_{r s}$ & $(22,1)(22,2)(23,1)(23,2)$ \\
$\widetilde{G}_{r s, 22}$ & $(31)(32)(33)$ \\
$\partial_{3} \widetilde{G}_{r s, 2}$ & $(11)(12)(22)$ \\
\end{tabular}

All components of one-order derivatives of Green's function has been presented in Zhang (2004) and Zhang and Chen (2006a). Based on these components, two-order derivatives of Green's function can be easily obtained by taking another derivative:

$$
\begin{aligned}
\widetilde{G}_{11,22}= & \frac{1}{4 \pi \mu}\left[a_{1}\left(I_{3,2}^{(0)}+I_{4,2}^{(0)}\right)+a_{2}\left(I_{1,2}^{(0)}+I_{2,2}^{(0)}\right)-a_{3}\left(I_{1,2}^{(1)}+I_{2,2}^{(1)}\right)-a_{4}\left(I_{3,2}^{(1)}+I_{4,2}^{(1)}\right)+a_{1,2}\left(I_{3}^{(0)}+I_{4}^{(0)}\right)+\right. \\
& \left.a_{2,2}\left(I_{1}^{(0)}+I_{2}^{(0)}\right)-a_{3,2}\left(I_{1}^{(1)}+I_{2}^{(1)}\right)-a_{4,2}\left(I_{3}^{(1)}+I_{4}^{(1)}\right)\right], \\
\widetilde{G}_{12,22}= & \frac{1}{4 \pi \mu}\left[a_{5}\left(I_{1,2}^{(0)}+I_{2,2}^{(0)}-I_{3,2}^{(0)}-I_{4,2}^{(0)}\right)-a_{6}\left(I_{1,2}^{(1)}+I_{2,2}^{(1)}-I_{3,2}^{(1)}+I_{4,2}^{(1)}\right)+a_{5,2}\left(I_{1}^{(0)}+I_{2}^{(0)}-I_{3}^{(0)}-I_{4}^{(0)}\right)-\right. \\
& \left.a_{6,2}\left(I_{1}^{(1)}+I_{2}^{(1)}-I_{3}^{(1)}+I_{4}^{(1)}\right)\right], \\
\widetilde{G}_{22,22}= & \frac{1}{4 \pi \mu}\left[a_{1}\left(I_{1,2}^{(0)}+I_{2,2}^{(0)}\right)+a_{2}\left(I_{3,2}^{(0)}+I_{4,2}^{(0)}\right)-a_{3}\left(I_{3,2}^{(1)}+I_{4,2}^{(1)}\right)-a_{4}\left(I_{1,2}^{(1)}+I_{2,2}^{(1)}\right)+a_{1,2}\left(I_{1}^{(0)}+I_{2}^{(0)}\right)+\right. \\
& \left.a_{2,2}\left(I_{3}^{(0)}+I_{4}^{(0)}\right)-a_{3,2}\left(I_{3}^{(1)}+I_{4}^{(1)}\right)-a_{4,2}\left(I_{1}^{(1)}+I_{2}^{(1)}\right)\right], \\
& \partial_{3} \widetilde{G}_{12,2}=\frac{1}{4 \pi \mu}\left[a_{5}\left(I_{1,3}^{(0)}+I_{2,3}^{(0)}-I_{3,3}^{(0)}-I_{4,3}^{(0)}\right)-a_{6}\left(I_{1,3}^{(1)}+I_{2,3}^{(1)}-I_{3,3}^{(1)}+I_{4,3}^{(1)}\right)\right],
\end{aligned}
$$




$$
\begin{gathered}
\partial_{3} \widetilde{G}_{31,2}=-\frac{\gamma_{1} \gamma_{2}}{4 \pi \mu}\left\{\frac{1}{r} I_{5,3}^{(0)}-I_{5,3}^{(1)}\right\}, \\
\partial_{3} \widetilde{G}_{32,2}=\frac{1}{4 \pi \mu}\left\{\frac{1-\gamma_{2}^{2}}{r} I_{5,3}^{(0)}+\gamma_{2}^{2} I_{5,3}^{(1)}\right\},
\end{gathered}
$$

where

$$
\begin{gathered}
a_{1}=\frac{2 \gamma_{2}\left(\gamma_{2}^{2}-1\right)}{r}, \quad a_{2}=\frac{2 \gamma_{1}^{2} \gamma_{2}}{r}, \quad a_{3}=\gamma_{1}^{2} \gamma_{2}, \\
a_{4}=\gamma_{2}^{3}, \quad a_{5}=\frac{\gamma_{1}\left(\gamma_{2}^{2}-1\right)}{r}, \quad a_{6}=\gamma_{1} \gamma_{2}^{2}, \\
a_{1,2}=\frac{2\left(\gamma_{2}^{2}-1\right)\left(4 \gamma_{2}^{2}-1\right)}{r^{2}}, \quad a_{2,2}=\frac{2 \gamma_{1}^{2}\left(4 \gamma_{2}^{2}-1\right)}{r^{2}}, \\
a_{3,2}=\frac{\gamma_{1}^{2}\left(3 \gamma_{2}^{2}-1\right)}{r}, \quad a_{4,2}=\frac{3 \gamma_{2}^{2}\left(\gamma_{2}^{2}-1\right)}{r} \\
a_{5,2}=\frac{4 \gamma_{1} \gamma_{2}\left(\gamma_{2}^{2}-1\right)}{r^{2}}, \quad a_{6,2}=\frac{\gamma_{1} \gamma_{2}\left(3 \gamma_{2}^{2}-1\right)}{r},
\end{gathered}
$$

with $\gamma_{\alpha}=\left(x_{\alpha}-\xi_{\alpha}\right) / r$ and $r=\sqrt{\left(x_{1}-\xi_{1}\right)^{2}+\left(x_{2}-\xi_{2}\right)^{2}}$. Detailed expressions for $I_{i}^{(\alpha-1)}, I_{i, j}^{(\alpha-1)}$, and $I_{5,3}^{(\alpha-1)}(\alpha=1,2$, $i=1,2,3,4$ and $j=1,2,3)$ are listed in equation (D1) in Appendix D.

For coplanar case, equation (25) can be greatly simplified. Since $\gamma_{1}=\operatorname{sgn}\left(x_{1}-\xi_{1}\right)$ and $\gamma_{2}=0$,

$$
\begin{aligned}
& a_{1}=a_{2}=a_{3}=a_{4}=a_{6}=0, \quad a_{5}=-\frac{1}{r} \operatorname{sgn}\left(x_{1}-\xi_{1}\right), \\
& a_{1,2}=-a_{2,2}=\frac{2}{r^{2}}, \quad a_{3,2}=-\frac{1}{r}, \quad a_{4,2}=a_{5,2}=a_{6,2},
\end{aligned}
$$

components of derivatives of Green's function in Table 1 can be reduced as

$$
\begin{gathered}
\widetilde{G}_{11,2}=\widetilde{G}_{12,1}=\widetilde{G}_{22,2}=\partial_{3} \widetilde{G}_{32}=\partial_{3} \widetilde{G}_{31,2}=0, \\
\widetilde{G}_{12,2}=\frac{\operatorname{sgn}\left(x_{1}-\xi_{1}\right)}{4 \pi \mu r} \int_{0}^{+\infty}\left(A_{S}^{(1)}-A_{T}^{(1)}\right) J_{2}(k r) \frac{1}{r} \mathrm{~d} k= \\
\frac{\operatorname{sgn}\left(x_{1}-\xi_{1}\right)}{16 \pi \mu} \int_{0}^{+\infty}\left(A_{S}^{(1)}-A_{T}^{(1)}\right)\left[J_{1}(k r)+J_{3}(k r)\right] \mathrm{d} k,(28.2 \\
\widetilde{G}_{22,1}=\frac{\operatorname{sgn}\left(x_{1}-\xi_{1}\right)}{4 \pi \mu r} \int_{0}^{+\infty}\left[\left(A_{S}^{(1)}-A_{T}^{(1)}\right) \frac{J_{2}(k r)}{k r}+\right. \\
\left.A_{T}^{(1)} J_{1}(k r)\right] \mathrm{d} k=\widetilde{G}_{12,2}+\frac{\operatorname{sgn}\left(x_{1}-\xi_{1}\right)}{4 \pi \mu} \int_{0}^{+\infty} A_{T}^{(1)} J_{1}(k r) \mathrm{d} k, \\
\widetilde{G}_{11.22}=\frac{\operatorname{sgn}\left(x_{1}-\xi_{1}\right)}{4 \pi \mu r} \int_{0}^{+\infty}\left[3\left(A_{S}^{(1)}-A_{T}^{(1)}\right) \frac{J_{2}(k r)}{k r}-\right.
\end{gathered}
$$

$$
\begin{gathered}
\left.A_{S}^{(1)} J_{1}(k r)\right] \mathrm{d} k=\frac{1}{8 \pi \mu} \int_{0}^{+\infty}\left\{\frac { 1 } { 4 } ( A _ { S } ^ { ( 1 ) } - A _ { T } ^ { ( 1 ) } ) \left[3 J_{0}(k r)+\right.\right. \\
\left.\left.4 J_{2}(k r)+J_{4}(k r)\right]-A_{S}^{(1)}\left[J_{0}(k r)+J_{2}(k r)\right]\right\} \mathrm{d} k .
\end{gathered}
$$

Substituting equations (25) and (28) into equations (C1) and (C2) in Appendix C, respectively, line or surface integration on derivatives of Green's function can be computed by applying proper numerical algorithm. After this, integral kernels in equations (19) and (23) can be evaluated by performing an inverse Fourier transform.

\section{Computation of the spontaneous rupture process}

Note that the Green's function itself is expressed as an oscillatory infinite integral on wave-number $k$ in frequency domain, another line or surface integration on such a function will be very time consuming. It is crucial to enhance the efficiency of computation of integral kernels (Zhang and Chen, 2006a). Otherwise, the BIEs derived above can not be performed practically at all. Fortunately, in the case of vertical fault, symmetry in the geometry can speed up the computation greatly. It is noted that in equations (D1) and (D2) in Appendix D, $x_{3}$ and $\xi_{3}$ only appears in exponential factors $\mathrm{e}_{P P}, \mathrm{e}_{S S}, \mathrm{e}_{S P}$ and $\mathrm{e}_{P S}$. Therefore, integrals on $\xi_{3}$ can be obtained analytically by applying the following result

$$
\int_{\xi_{3}^{m}}^{\xi_{3}^{m+1}} \mathrm{e}^{-\left(a x_{3}+b \xi_{3}\right)} \mathrm{d} \xi_{3}=-\frac{1}{b} \mathrm{e}^{-a x_{3}}\left(\mathrm{e}^{-b \xi_{3}^{m+1}}-\mathrm{e}^{-b \xi_{3}^{m}}\right),
$$

and multiple-integrals is reduce by one-order, which makes the computation of integral kernels much more efficient. By applying the algorithm based on detailed analysis on integrand (Zhang and Chen, 2006a), the time required to compute integral kernels is fairly acceptable.

Only geometrical quantities are involved in the integral kernels, which implies that as long as the geometry of the fault system is known, integral kernels can be computed in advance. This is an important advantage of the BIEM. When the CFL value (e.g., Madariaga et al, 1998 ) is less than 0.5 , only the source points in collocation element (i.e. the source point and observation point are in the same element) at current time step will contribute to the stress component, and slip rate and stress component at past time step are all known. Therefore, for observation point at a given position ( $i$ and $j$ ) and time $(k)$, there are three unknowns in equation (24): $\tau_{21}^{i j k}$, 
$\tau_{22}^{i j k}$ and $V_{1}^{\prime j k}$. Two equations are not enough to solve three unknowns. To solve a spontaneous rupture propagation problem, another equation is needed. That is the so-called friction law based on rock experiments. A possible choice is the slip-weakening friction law, which is widely used in the dynamic modelings of earthquake source (e.g., Ida, 1972; Andrew, 1976; Day, 1982; Olsen et al, 1997; Fukuyama and Madariaga, 1998):

$$
\tau(\Delta u)=\tau_{\mathrm{r}}+\left(\tau_{\mathrm{p}}-\tau_{\mathrm{r}}\right)\left(1-\frac{\Delta u}{D_{\mathrm{c}}}\right) H\left(D_{\mathrm{c}}-\Delta u\right),
$$

where $\tau$ is the shear stress, $\Delta u$ is the slip, $D_{c}$ is the critical weakening displacement, $\tau_{\mathrm{p}}$ and $\tau_{\mathrm{r}}$ are peak strength and residual strength, respectively. Solving equations (24) and (30) will produce the final solution.

However, it is difficult to directly assign the values of the stress required, such as initial field $\tau_{0}$, the peak strength $\tau_{\mathrm{p}}$ and the residual strength $\tau_{\mathrm{r}}$. We follow Aochi and Madariaga (2003) to infer these parameters indirectly by introducing other parameters which are possible to constrain by other means.

Considering the effect of the normal stress, $\tau_{\mathrm{p}}$ and $\tau_{\mathrm{r}}$ are not assigned in advance, but connected with the normal stress through static and dynamic frictional coefficients $\mu_{\mathrm{s}}$ and $\mu_{\mathrm{d}}$ (Aochi and Madariaga, 2003):

$$
\tau_{\mathrm{p}}=\sigma_{0}+\mu_{\mathrm{s}} \sigma_{\mathrm{n}}, \quad \tau_{\mathrm{r}}=\mu_{\mathrm{d}} \sigma_{\mathrm{n}},
$$

where $\sigma_{0}$ is cohesive force, and $\sigma_{\mathrm{n}}$ is the applied normal stress on the fault. Suppose that a uniform external load (remote tectonic stress) is applied over all the rupture area. The external principal stress $\sigma_{1}, \sigma_{2}$ and $\sigma_{3}$ are unknown. The intermediate stress $\sigma_{2}$ is supposed to be vertical at all depth in order to permit a strike-slip movement, and it is equal to lithostatic pressure $P$. For simplicity, we suppose $\sigma_{2}=\left(\sigma_{1}+\sigma_{3}\right) / 2$. Letting $\Delta \sigma=\left(\sigma_{1}-\sigma_{3}\right) / 2$, then the principal stresses are

$$
\sigma_{1}=P+\Delta \sigma, \quad \sigma_{2}=P, \quad \sigma_{3}=P-\Delta \sigma .
$$

Suppose that the direction of the subfault $\Sigma_{\mathrm{s}}$, from which the rupture starts (the angle between $\Sigma_{\mathrm{s}}$ and the maximum principal stress $\sigma_{1}$ is $\Phi_{0}$, see Figure $4 \mathrm{a}$ ), is consistent with that expected by the Mohr-Coulomb criterion (Figure 4b). Define parameter $R$ as

$$
R=\frac{\Delta \tau}{\Delta \tau_{b}}=\frac{\tau_{0}-\tau_{r}}{\tau_{p}-\tau_{r}}
$$

Now the problem is, given $\mu_{s}, \mu_{d}, \sigma_{0}, P$ and $R$, we determine the initial field on subfault $\Sigma_{q}$, say $\tau_{0}^{(q)}$ and $\sigma_{\mathrm{n} 0}^{(q)}, \tau_{\mathrm{p}}^{(q)}$, and $\tau_{\mathrm{r}}^{(q)}$.

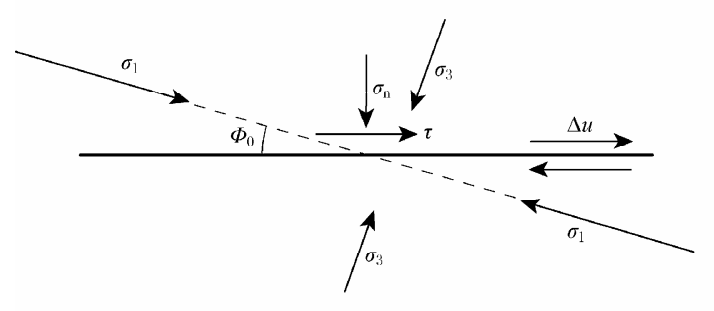

(a)

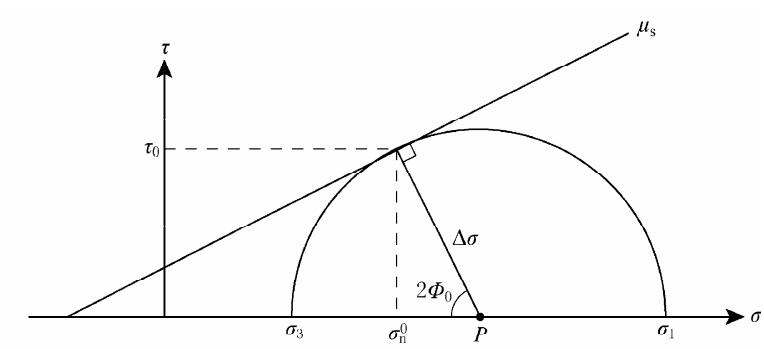

(b)

Figure 4 Schematic illustration of the assumed initial situation (modified from Aochi and Madariaga, 2003). (a) Relation between maximum and minimum principal stress $\sigma_{1}$ and $\sigma_{2}$ with respect to the subfault $\Sigma_{s}$, from which the rupture starts. (b) MohrCoulomb criterion for determining the angle $\Phi_{0}$ between $\Sigma_{s}$ and $\sigma_{1}$. Detailed explanations of each parameter are given in the text.

First, let we determine the initial field $\tau_{0}^{(s)}$ and $\sigma_{\mathrm{n} 0}^{(s)}$, and $\tau_{\mathrm{p}}^{(s)}$, and $\tau_{\mathrm{r}}^{(s)}$ on subfault $\Sigma_{s}$. In Figure $4 \mathrm{~b}$, straight line denotes the Coulomb fracture line, whose rate of slope is $\mu_{\mathrm{s}}$. When rupture starts, this line is tangent to the Mohr circle. Therefore,

$$
\tan \left(\frac{\pi}{2}-2 \Phi_{0}\right)=\mu_{\mathrm{s}},
$$

and $\Phi_{0}$ can be estimated as

$$
\Phi_{0}=\frac{\pi}{4}-\frac{1}{2} \tan ^{-1} \mu_{\mathrm{s}} .
$$

From Figure $4 b$, we have

$$
\left(\tau_{0}^{(s)}\right)^{2}+\left(\sigma_{\mathrm{n} 0}^{(s)}-P\right)^{2}=\Delta \sigma^{2},
$$

where

$$
\left\{\begin{array}{l}
\tau_{0}^{(s)}=\Delta \sigma \cdot \sin \left(2 \Phi_{0}\right), \\
\sigma_{\mathrm{n} 0}^{(s)}=P-\Delta \sigma \cdot \cos \left(2 \Phi_{0}\right),
\end{array}\right.
$$


According to equation (31), we have

$$
\left\{\begin{array}{l}
\tau_{\mathrm{p}}^{(s)}=\sigma_{0}+\mu_{\mathrm{s}} \sigma_{\mathrm{n} 0}^{(s)}=\sigma_{0}+\mu_{\mathrm{s}} P-\mu_{\mathrm{s}} \Delta \sigma \cdot \cos \left(2 \Phi_{0}\right), \\
\tau_{\mathrm{r}}^{(s)}=\mu_{\mathrm{d}} \sigma_{\mathrm{n} 0}^{(s)}=\mu_{\mathrm{d}} P-\mu_{\mathrm{d}} \Delta \sigma \cdot \cos \left(2 \Phi_{0}\right) .
\end{array}\right.
$$

Substituting equations (37) and (38) into equation (33), we get

$$
\begin{gathered}
R= \\
\frac{\Delta \sigma \cdot \sin \left(2 \Phi_{0}\right)-\mu_{\mathrm{d}} P+\mu_{\mathrm{d}} \Delta \sigma \cdot \cos \left(2 \Phi_{0}\right)}{\sigma_{0}+\mu_{\mathrm{s}} P-\mu_{\mathrm{s}} \Delta \sigma \cdot \cos \left(2 \Phi_{0}\right)-\mu_{\mathrm{d}} P+\mu_{\mathrm{d}} \Delta \sigma \cdot \cos \left(2 \Phi_{0}\right)}
\end{gathered}
$$

from which $\Delta \sigma$ can be evaluated as

$$
\begin{gathered}
\Delta \sigma= \\
\frac{\mu_{\mathrm{d}} P+R\left[\sigma_{0}+\left(\mu_{\mathrm{s}}-\mu_{\mathrm{d}}\right) P\right]}{\sin \left(2 \Phi_{0}\right)+\mu_{\mathrm{d}} \cos \left(2 \Phi_{0}\right)+R\left(\mu_{\mathrm{s}}-\mu_{\mathrm{d}}\right) \cos \left(2 \Phi_{0}\right)} .
\end{gathered}
$$

Given $\Delta \sigma$ in equation (40), $\tau_{0}^{(s)}, \sigma_{\mathrm{n} 0}^{(s)}, \tau_{\mathrm{p}}^{(s)}$, and $\tau_{\mathrm{r}}^{(s)}$ for subfault $\Sigma_{s}$ can be easily obtained by equations (37) and (38).

For other subfaults $\Sigma_{q}(q \neq s), \tau_{0}^{(q)}, \sigma_{\mathrm{n} 0}^{(q)}$ can be simply estimated through Mohr circle as follows. Let the angle between $\Sigma_{q}$ and the maximum principal stress $\sigma_{1}$ be $\Phi_{q}$. From Figure $5, \tau_{0}^{(q)}$ and $\sigma_{\mathrm{n} 0}^{(q)}$ can be written as

$$
\left\{\begin{array}{l}
\tau_{0}^{(q)}=\Delta \sigma \cdot \sin \left(2 \Phi_{q}\right) \\
\sigma_{\mathrm{n} 0}^{(q)}=P-\Delta \sigma \cdot \cos \left(2 \Phi_{q}\right) .
\end{array}\right.
$$

From equation (31), $\tau_{\mathrm{p}}^{(q)}$ and $\tau_{\mathrm{r}}^{(q)}$ are obtained as

$$
\left\{\begin{array}{l}
\tau_{\mathrm{p}}^{(q)}=\sigma_{0}+\mu_{\mathrm{s}} \sigma_{\mathrm{n} 0}^{(q)}=\sigma_{0}+\mu_{\mathrm{s}} P-\mu_{\mathrm{s}} \Delta \sigma \cdot \cos \left(2 \Phi_{q}\right), \\
\tau_{\mathrm{r}}^{(q)}=\mu_{\mathrm{d}} \sigma_{\mathrm{n} 0}^{(s)}=\mu_{\mathrm{d}} P-\mu_{\mathrm{d}} \Delta \sigma \cdot \cos \left(2 \Phi_{q}\right) .
\end{array}\right.
$$

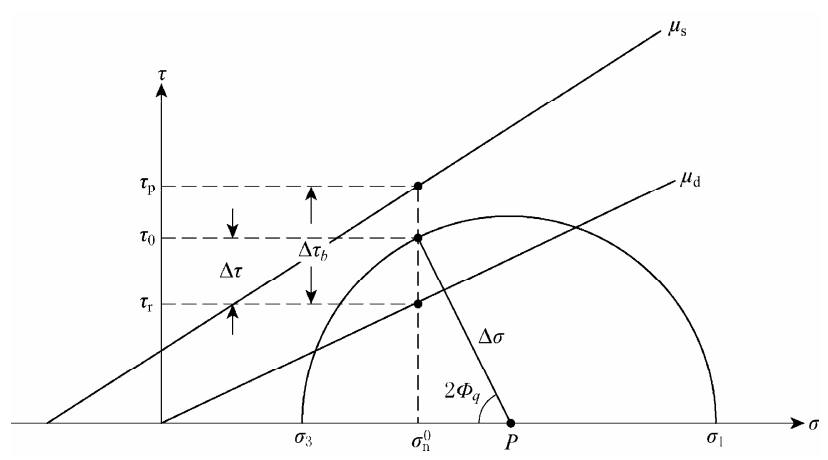

Figure 5 Mohr circle for determination of initial stress $\tau_{0}^{(q)}$, $\sigma_{\mathrm{n} 0}^{(q)}$ on subfault $\Sigma_{q}$. Detailed explanations of each parameter are given in the text.

\section{Conclusions}

In this article, we derived the BIEs as applied in the modeling of vertical complex fault system embedded in half-space. The BIEs are composed of two parts, contribution from full-space and that from free surface. The former is exactly the same as that obtained based on full-space Green's function (Aochi et al, 2000). We focus on evaluating the effect of free surface. This is accomplished by solving the non-coplanar part and co-planar part, respectively. Special care is paid to practical computation of the integral kernels and initial field. As stated by Tada (2009), advantages of the presented BIEM over other numerical methods include (1) easy adaptability to problems that have complex fault geometry, (2) suitability to problems that involve unbounded domains, and (3) separation of the computation of integral kernels and rupture processes. Although the difficulty of dealing with inhomogeneous media should be counted as one of its principal disadvantages, this work pushes forward the BIEM to a more real medium model.

The proposed BIEs need validation and numerical examples to show the capacity and relevance. These are works left to near future.

Acknowledgments This work is jointly supported by the President Fund of GUCAS (No. O85101CM03) and National Natural Science Foundation of China (Nos. 90715019 and 40821062), and partially by National Basic Research Program of China (No.2004CB418404).

\section{References}

Aki K and Richards P G (2002). Quantitative Seismology. The 2nd edition. University Science Books, Sausalito, California, 72.

Andrews D J (1976). Rupture velocity of plane strain shear cracks. J Geophys Res 81: 5679-5687.

Aochi H and Fukuyama E (2002). Three-dimensional nonplanar simulation of the 1992 Landers earthquake. J Geophys Res 107: 10.1029/2000JB000061.

Aochi H and Madariaga R (2003). The 1999 'Izmit, Turkey, earthquake: Nonplanar fault structure, dynamic rupture process, and strong ground motion. Bull Seism Soc Amer 93: 1249-1 266.

Aochi H, Fukuyama E and Matsu'ura M (2000). Spontaneous rupture propagation on a non-planar fault in 3-D elastic medium. Pure Appl Geophys 157: 2003-2 027.

Aochi H, Madariaga R and Fukuyama E (2002). Effect of normal stress during rupture propagation along nonplanar faults. $J$ Geophys Res 107: 10.1029/2001JB000500.

Aochi H, Scotti O and Berge-Thierry C (2005). Dynamic transfer of rupture across differently oriented segments in a complex 3-D fault system. Geophys Res Lett 32: L21304, doi:10.1029/2005GL024158.

Bouchon M and Streiff D (1997). Propagation of a shear crack on a nonplanar 
fault: A method of calculation. Bull Seism Soc Amer 87: 61-66.

Chen X F and Zhang H M (2006). Modelling rupture dynamics of a planar fault in 3-D half space by boundary integral equation method: An overview. Pure Appl Geophys 163: 267-299.

Day S (1982). Three-dimensional simulation of spontaneous rupture: The effect of nonuniform prestress. Bull Seism Soc Amer 72: 1 881-1 902.

Fukuyama E and Madariaga R (1998). Rupture dynamics of a plannar fault in a 3D elastic medium: Rate-and slip-weakening friction. Bull Seism Soc Amer 88: 1-17.

Harris R A, Archuleta R J and Day S M (1991). Fault steps and the dynamic rupture process: 2-D numerical simulations of a spontaneously propagating shear fracture. Geophys Res Lett 18: 893-896.

Harris R A and Day S M (1993). Dynamics of fault interaction: parallel strike-slip faults. $J$ Geophys Res 98: 4 461-4 472.

Harris R A and Day S M (1999). Dynamic 3-D simulations of earthquakes on an echelon faults. Geophys Res Lett 26: 2089-2 092.

Ida Y (1972). Cohesive force across the tip of a longitudinal-shear crack and Griffith's specific surface energy. J Geophys Res 77: 3796-3 805.

Kame N and Yamashita T (1997). Dynamic nucleation process of shallow earthquake faulting in a fault zone. Geophys J Int 128: 204-216.

Kame N and Yamashita T (1999). A new light on arresting mechanism of dynamic earthquake faulting. Geophys Res Lett 26: 1997-2 000.

Koller M, Bonnet M and Madariaga R (1992). Modelling of dynamical crack propagation using time-domain boundary integral equations. Wave Motion 16: $339-366$.

Lin A M, Fu B H, Guo J M, Zeng Q L, Dang G M, He W G and Zhao Y (2002). Co-seismic strike-slip and rupture length produced by the $2001 M_{\mathrm{S}} 8.1$ Central Kunlun earthquake. Science 296: 2015-2 017.

Madariaga R, Olsen K B and Archuleta R J (1998). Modeling dynamic rupture in a 3D earthquake fault model. Bull Seism Soc Amer 88: 1 182-1 197.

Nielsen S (1998). Free surface effects on the propagation of dynamic rupture. Geophys Res Lett 25: 125-128.

Oglesby D D, Archuleta R J and Nielson S (2000). The three-dimensional dynamics of dipping faults. Bull Seism Soc Amer 90: 616-628.
Olsen K B, Madariaga R, and Archuleta R J (1997). Three dimensional dynamic simulations of the 1992 Landers earthquake. Science 278: 834-838.

Quin H (1990). Dynamic stress drop and rupture dynamics of the October 15, 1979 Imperial Valley, California, earthquake. Tectonophysics 175: 93-117.

Shin T C and Teng T L (2001). An overview of the 1999 Chi-Chi, Taiwain, earthquake. Bull Seism Soc Amer 91: 895-914.

Tada T (2009). Boundary integral equation method for earthquake rupture dynamics. Chapter 9. In: Fukuyama E. Fault-zone Properties and Earthquake. Elsevier Academic Press, 217.

Tada T, Fukuyama E and Madariaga R (2000). Non-hypersingular boundary integral equations for 3-D non-planar crack dynamics. Comput Mech 25: 613-626.

Tada T and Yamashita T (1997). Non-hypersingular boundary integral equations for two-dimensional non-planar crack analysis. Geophys $J$ Int 130: 269-282.

Xu X W, Wen X Z, Ye J Q, Ma B Q, Chen J, Zhou R J, He H L, Tian Q J, He Y L, Wang Z C, Sun Z M, Feng X J, Yu G H, Chen L C, Chen G H, Yu S E, Ran Y K, Li X G, Li C X and An Y F (2008). The $M_{\mathrm{S}} 8.0$ Wenchuan earthquake surface ruptures and its seismogenic structure. Seismology and Geology 30: 597-629 (in Chinese with English abstract).

Yamashita T and Fukuyama E (1996). Apparent critical slip displacement cased by the existence of a fault zone. Geophys J Int 125: 459-472.

Zhang H M (2004). Theoretical Study on the Spontaneous Propagation of 3-D Seismic Rupture on a Planar Fault in Half-space. [Ph D Dissertation]. Peking University, Beijing.

Zhang H M and Chen X F (2006a). Dynamic rupture on a planar fault in three-dimensional half space. I. Theory. Geophys J Int 164: 633-652.

Zhang H M and Chen X F (2006b). Dynamic rupture on a planar fault in three-dimensional half space. II. Validations and numerical experiments. Geophys J Int 167: 917-932.

Zhang H M and Chen X F (2009). Equivalence of the Green's function for a full-space to the direct-wave contributions for a half-space and a layered half-space. Bull Seism Soc Amer 99: 454-461.

\section{Appendix A Regularized integrals}

For non-coplanar part,

$$
\begin{aligned}
& \iint_{\Sigma_{q}^{\prime}} \Delta \widetilde{u}_{\alpha} \partial_{1} \widetilde{G}_{i j, 1} \mathrm{~d} \xi_{1} \mathrm{~d} \xi_{3}=\iint_{\Sigma_{q}^{\prime}}\left(\Delta \widetilde{u}_{\alpha, 1}-\eta \Delta \widetilde{u}_{\alpha, 2}\right)\left(\widetilde{G}_{i, j, 1}+\widetilde{G}_{i j, 2}\right) \mathrm{d} \xi_{1} \mathrm{~d} \xi_{3}-\eta^{2} \iint_{\Sigma_{q}} \Delta \widetilde{u}_{\alpha} \widetilde{G}_{i j, 22} \mathrm{~d} \xi_{1} \mathrm{~d} \xi_{3}, \\
& \iint_{\Sigma_{q}} \Delta \widetilde{u}_{\alpha} \partial_{1} \widetilde{G}_{i j, 2} \mathrm{~d} \xi_{1} \mathrm{~d} \xi_{3}=\iint_{\Sigma_{q}} \Delta \widetilde{u}_{\alpha} \partial_{2} \widetilde{G}_{i j, 1} \mathrm{~d} \xi_{1} \mathrm{~d} \xi_{3}=\iint_{\Sigma_{q}^{\prime}}\left(\Delta \widetilde{u}_{\alpha, 1}-\eta \Delta \widetilde{u}_{\alpha, 2}\right) \widetilde{G}_{i j, 2} \mathrm{~d} \xi_{1} \mathrm{~d} \xi_{3}-\eta \iint_{\Sigma_{q}} \Delta \widetilde{u}_{\alpha} \widetilde{G}_{i j, 22} \mathrm{~d} \xi_{1} \mathrm{~d} \xi_{3}, \\
& \iint_{\Sigma_{q}} \Delta \widetilde{u}_{\alpha} \partial_{\beta} \widetilde{G}_{i j, 3} \mathrm{~d} \xi_{1} \mathrm{~d} \xi_{3}=\iint_{\Sigma_{q}^{\prime}} \Delta \widetilde{u}_{\alpha, 3} \widetilde{G}_{i j, \beta} \mathrm{d} \xi_{1} \mathrm{~d} \xi_{3}, \\
& \iint_{\Sigma_{q}^{\prime}} \Delta \widetilde{u}_{\alpha} \partial_{2} \widetilde{G}_{i j, 2} \mathrm{~d} \xi_{1} \mathrm{~d} \xi_{3}=-\iint_{\Sigma_{q}} \Delta \widetilde{u}_{\alpha} \widetilde{G}_{i j, 22} \mathrm{~d} \xi_{1} \mathrm{~d} \xi_{3}, \\
& \iint_{\Sigma_{q}^{\prime}} \Delta \widetilde{u}_{\alpha} \partial_{3} \widetilde{G}_{i j, 1} \mathrm{~d} \xi_{1} \mathrm{~d} \xi_{3}=-\iint_{\Sigma_{q}}\left(\Delta \widetilde{u}_{\alpha, 1}-\eta \Delta \widetilde{u}_{\alpha, 2}\right) \partial_{3} \widetilde{G}_{i j} \mathrm{~d} \xi_{1} \mathrm{~d} \xi_{3}+\eta \iint_{\Sigma_{q}} \Delta \widetilde{u}_{\alpha} \partial_{3} \widetilde{G}_{i j, 2} \mathrm{~d} \xi_{1} \mathrm{~d} \xi_{3}, \\
& \iint_{\Sigma_{q}} \Delta \widetilde{u}_{\alpha} \partial_{3} \widetilde{G}_{i j, 3} \mathrm{~d} \xi_{1} \mathrm{~d} \xi_{3}=-\iint_{\Sigma_{q}} \Delta \widetilde{u}_{\alpha, 3} \partial_{3} \widetilde{G}_{i j} \mathrm{~d} \xi_{1} \mathrm{~d} \xi_{3} .
\end{aligned}
$$

and for coplanar part,

$$
\begin{aligned}
& \iint_{\Sigma_{q_{0}}} \Delta \widetilde{u}_{1} \partial_{1} \widetilde{G}_{i j, \alpha} \mathrm{d} \xi_{1} \mathrm{~d} \xi_{3}=\iint_{\Sigma_{q_{0}}} \Delta \widetilde{u}_{1,1} \widetilde{G}_{i j, \alpha} \mathrm{d} \xi_{1} \mathrm{~d} \xi_{3}, \\
& \iint_{\Sigma_{q_{0}}} \Delta \widetilde{u}_{1} \partial_{2} \widetilde{G}_{i j, 1} \mathrm{~d} \xi_{1} \mathrm{~d} \xi_{3}=\iint_{\Sigma_{q_{0}}} \Delta \widetilde{u}_{1,1} \widetilde{G}_{i j, 2} \mathrm{~d} \xi_{1} \mathrm{~d} \xi_{3}, \\
& \iint_{\Sigma_{q_{0}}} \Delta \widetilde{u}_{1} \partial_{2} \widetilde{G}_{i j, 2} \mathrm{~d} \xi_{1} \mathrm{~d} \xi_{3}=-\iint_{\Sigma_{q_{0}}} \Delta \widetilde{u}_{1} \widetilde{G}_{i j, 22} \mathrm{~d} \xi_{1} \mathrm{~d} \xi_{3},
\end{aligned}
$$




$$
\iint_{\Sigma_{q_{0}}} \Delta \widetilde{u}_{1} \partial_{3} \widetilde{G}_{i j, 1} \mathrm{~d} \xi_{1} \mathrm{~d} \xi_{3}=-\iint_{\Sigma_{q_{0}}} \Delta \widetilde{u}_{1,1} \partial_{3} \widetilde{G}_{i j} \mathrm{~d} \xi_{1} \mathrm{~d} \xi_{3}
$$

Appendix B Expressions for $U_{\alpha i j k}^{\beta}, V_{\alpha i j k}^{\beta}, W_{\alpha i j k}^{\beta}, X_{i j k}, Y_{i j k}$ and $Z_{i j k}$

For non-coplanar part,

$$
\begin{gathered}
U_{\alpha i j k}^{\mathrm{I}}\left(\left.\boldsymbol{x}^{P}\right|_{\Sigma_{q_{0}}}, \omega\right)=\mu^{2} \sum_{q=1 \wedge q \neq q_{0}}^{Q} \iint_{\Sigma_{q}^{\prime}}\left\{\left[\Delta \widetilde{u}_{\alpha, 1}(\boldsymbol{\xi}, \omega)-\eta \Delta \widetilde{u}_{\alpha, 2}(\boldsymbol{\xi}, \omega)\right] \cdot \widetilde{G}_{i j, k}\left(\left.\boldsymbol{x}^{P}\right|_{\Sigma_{q_{0}}}, \boldsymbol{\xi}, \omega\right)\right\}_{\xi_{2}=L_{q}\left(\xi_{1}\right)} \mathrm{d} \xi_{1} \mathrm{~d} \xi_{3}, \\
U_{\alpha i j k}^{\mathrm{II}}\left(\left.\boldsymbol{x}^{P}\right|_{\Sigma_{q_{0}}}, \omega\right)=\mu^{2} \sum_{q=1 \wedge q \neq q_{0}}^{Q} \iint_{\Sigma_{q}^{\prime}}\left\{\Delta \widetilde{u}_{\alpha, 3}(\xi, \omega) \widetilde{G}_{i j, k}\left(\left.\boldsymbol{x}^{P}\right|_{\Sigma_{q_{0}}}, \boldsymbol{\xi}, \omega\right)\right\}_{\xi_{2}=L_{q}\left(\xi_{1}\right)} \mathrm{d} \xi_{1} \mathrm{~d} \xi_{3}, \\
V_{\alpha i j}^{\mathrm{I}}\left(\left.\boldsymbol{x}^{P}\right|_{\Sigma_{q_{0}}}, \omega\right)=\mu^{2} \sum_{q=1 \wedge q \neq q_{0}}^{Q} \iint_{\Sigma_{q}^{\prime}}\left\{\left[\Delta \widetilde{u}_{\alpha, 1}(\boldsymbol{\xi}, \omega)-\eta \Delta \widetilde{u}_{\alpha, 2}(\boldsymbol{\xi}, \omega)\right] \cdot \partial_{3} \widetilde{G}_{i j}\left(\left.\boldsymbol{x}^{P}\right|_{\Sigma_{q_{0}}}, \boldsymbol{\xi}, \omega\right)\right\}_{\xi_{2}=L_{q}\left(\xi_{1}\right)} \mathrm{d} \xi_{1} \mathrm{~d} \xi_{3}, \\
V_{\alpha i j}^{\mathrm{II}}\left(\left.\boldsymbol{x}^{P}\right|_{\Sigma_{q_{0}}}, \omega\right)=\mu^{2} \sum_{q=1 \wedge q \neq q_{0}}^{Q} \iint_{\Sigma_{q}^{\prime}}\left\{\Delta \widetilde{u}_{\alpha, 3}(\boldsymbol{\xi}, \omega) \partial_{3} \widetilde{G}_{i j}\left(\left.\boldsymbol{x}^{P}\right|_{\Sigma_{q_{0}}}, \boldsymbol{\xi}, \omega\right)\right\}_{\xi_{2}=L_{q}\left(\xi_{1}\right)} \mathrm{d} \xi_{1} \mathrm{~d} \xi_{3}, \\
W_{\alpha i j}^{\mathrm{I}}\left(\left.\boldsymbol{x}^{P}\right|_{\Sigma_{q_{0}}}, \omega\right)=\mu^{2} \sum_{q=1 \wedge q \neq q_{0}}^{Q} \iint_{\Sigma_{q}^{\prime}}\left\{\Delta \widetilde{u}_{\alpha}(\boldsymbol{\xi}, \omega) \widetilde{G}_{i j, 22}\left(\left.\boldsymbol{x}^{\mathrm{P}}\right|_{\Sigma_{q_{0}}}, \boldsymbol{\xi}, \omega\right)\right\}_{\xi_{2}=L_{q}\left(\xi_{1}\right)} \mathrm{d} \xi_{1} \mathrm{~d} \xi_{3}, \\
W_{\alpha i j}^{\mathrm{II}}\left(\left.\boldsymbol{x}^{P}\right|_{\Sigma_{q_{0}}}, \omega\right)=\mu^{2} \sum_{q=1 \wedge q \neq q_{0}}^{Q} \iint_{\Sigma_{q}^{\prime}}\left\{\Delta \widetilde{u}_{\alpha}(\boldsymbol{\xi}, \omega) \partial_{3} \widetilde{G}_{i j, 2}\left(\left.\boldsymbol{x}^{\mathrm{P}}\right|_{\Sigma_{q_{0}}}, \boldsymbol{\xi}, \omega\right)\right\}_{\xi_{2}=L_{q}\left(\xi_{1}\right)} \mathrm{d} \xi_{1} \mathrm{~d} \xi_{3},
\end{gathered}
$$

and for coplanar part,

$$
\begin{gathered}
X_{i j k}\left(\left.\boldsymbol{x}^{P}\right|_{\Sigma_{q_{0}}}, \omega\right)=\mu^{2} \iint_{\Sigma_{q_{0}}}\left\{\Delta \widetilde{u}_{1,1}(\xi, \omega) \widetilde{G}_{i j, k}\left(\left.\boldsymbol{x}^{P}\right|_{\Sigma_{q 0}}, \boldsymbol{\xi}, \omega\right)\right\}_{\xi_{2}=0} \mathrm{~d} \xi_{1} \mathrm{~d} \xi_{3}, \\
Y_{i j}\left(\left.\boldsymbol{x}^{P}\right|_{\Sigma_{q_{0}}}, \omega\right)=\mu^{2} \iint_{\Sigma_{q_{0}}}\left\{\Delta \widetilde{u}_{1,1}(\xi, \omega) \partial_{3} \widetilde{G}_{i j}\left(\left.\boldsymbol{x}^{P}\right|_{\Sigma_{q_{0}}}, \boldsymbol{\xi}, \omega\right)\right\}_{\xi_{2}=0} \mathrm{~d} \xi_{1} \mathrm{~d} \xi_{3}, \\
Z_{i j}^{\mathrm{I}}\left(\left.\boldsymbol{x}^{P}\right|_{\Sigma_{q_{0}}}, \omega\right)=\mu^{2} \iint_{\Sigma_{q_{0}}}\left\{\Delta \widetilde{u}_{1,1}(\xi, \omega) \widetilde{G}_{i j, 22}\left(\left.\boldsymbol{x}^{P}\right|_{\Sigma_{q_{0}}}, \boldsymbol{\xi}, \omega\right)\right\}_{\xi_{2}=0} \mathrm{~d} \xi_{1} \mathrm{~d} \xi_{3}, \\
Z_{i j}^{\mathrm{II}}\left(\left.\boldsymbol{x}^{P}\right|_{\Sigma_{q_{0}}}, \omega\right)=\mu^{2} \iint_{\Sigma_{q_{0}}}\left\{\Delta \widetilde{u}_{1,1}(\boldsymbol{\xi}, \omega) \partial_{3} \widetilde{G}_{i j, 2}\left(\left.\boldsymbol{x}^{P}\right|_{\Sigma_{q_{0}}}, \boldsymbol{\xi}, \omega\right)\right\}_{\xi_{2}=0} \mathrm{~d} \xi_{1} \mathrm{~d} \xi_{3} .
\end{gathered}
$$

Appendix C Expressions for $D_{r s t}^{\beta: i j ; l m}, E_{r s}^{\beta: i j ; l m}, F_{r s t}^{\beta: i j: l m}, I_{r s t}^{i j: l m}, J_{r s}^{i j ; l m}$ and $K_{r s}^{\beta: i j ; l m}$

For non-coplanar part,

$$
\begin{aligned}
& D_{r s t}^{\mathrm{I} i j ; l m}(\omega)=\left.\int_{\xi_{3}^{\prime m}}^{\xi_{3}^{\prime m+1}} \widetilde{G}_{r s, t}\left(0,0, x_{3}^{j}, \xi_{1}, L_{q}\left(\xi_{1}\right), \xi_{3} ; \omega\right)\right|_{\xi_{1}=\xi_{1}^{i} ; l} ^{\xi_{1}=\xi_{1}^{i j l+1}} \mathrm{~d} \xi_{3}, \\
& D_{r s t}^{\mathrm{II} i j ; l m}(\omega)=\left.\int_{\xi_{1}^{i ; l}}^{\xi_{1}^{i j l+1}} \widetilde{G}_{r s, t}\left(0,0, x_{3}^{j}, \xi_{1}, L_{q}\left(\xi_{1}\right), \xi_{3} ; \omega\right)\right|_{\xi_{3}=\xi_{3}^{\prime m}} ^{\xi_{3}=\xi_{3}^{\prime m+1}} \mathrm{~d} \xi_{1}, \\
& E_{r s}^{\mathrm{I}: i j: l m}(\omega)=\left.\int_{\xi_{3}^{\prime m}}^{\xi_{3}^{\prime m+1}} \partial_{3} \widetilde{G}_{r s}\left(0,0, x_{3}^{j}, \xi_{1}, L_{q}\left(\xi_{1}\right), \xi_{3} ; \omega\right)\right|_{\xi_{1}=\xi_{1}^{i, l}} ^{\substack{\xi_{1}=\xi_{1}^{i l l} \\
y_{1}}} \mathrm{~d} \xi_{3},
\end{aligned}
$$

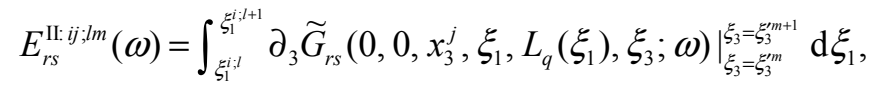

$$
\begin{aligned}
& F_{r s}^{\mathrm{I}: i j: l m}(\omega)=\int_{\xi_{3}^{m}}^{\xi_{3}^{m+1}} \int_{\xi_{1}^{i l l}}^{\xi_{1}^{i ! l+1}} \widetilde{G}_{r s, 22}\left(0,0, x_{3}^{j}, \xi_{1}, L_{q}\left(\xi_{1}\right), \xi_{3} ; \omega\right) \mathrm{d} \xi_{1} \mathrm{~d} \xi_{3},
\end{aligned}
$$




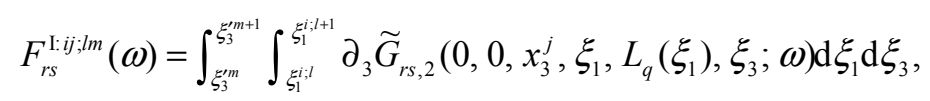

where $\xi_{1}^{i ; l}=v_{2} \xi_{1}^{\prime l}-v_{1}\left(\xi_{2}^{\prime}\right)_{O}-v_{2}\left(\xi_{1}^{\prime}\right)_{O}$, and for coplanar part,

$$
\begin{aligned}
& I_{r s t}^{i j: l m}(\omega)=\left.\int_{\xi_{3}^{\prime \prime m}}^{\xi_{3}^{\prime m+1}} \widetilde{G}_{r s, t}\left(0,0, x_{3}^{j}, \xi_{1}, 0, \xi_{3} ; \omega\right)\right|_{\xi_{1}=\xi_{1}^{i}} ^{\xi_{1}=\xi_{1}^{i: l t l}} \mathrm{~d} \xi_{3}, \\
& J_{r s}^{i j / l m}(\omega)=\left.\int_{\xi_{3}^{\prime m}}^{\xi_{3}^{\prime m+1}} \partial_{3} \widetilde{G}_{r s}\left(0,0, x_{3}^{j}, \xi_{1}, 0, \xi_{3} ; \omega\right)\right|_{\substack{\xi_{1}=\xi_{1} \\
\xi_{1}=\xi_{1}^{i, l}}} ^{\substack{i, l+1 \\
g^{\prime}}} \mathrm{d} \xi_{3}, \\
& K_{r s}^{\mathrm{I} i: j ; l m}(\omega)=\int_{\xi_{3}^{m m}}^{\xi_{3}^{\xi^{m+1}}} \int_{\xi_{1}: t}^{\xi_{1} \xi_{1} l t+1} \widetilde{G}_{r s, 22}\left(0,0, x_{3}^{j}, \xi_{1}, 0, \xi_{3} ; \omega\right) \mathrm{d} \xi_{1} \mathrm{~d} \xi_{3}, \\
& K_{r s}^{\mathrm{II}: i j: l m}(\omega)=\int_{\xi_{3}^{m}}^{\xi_{3}^{m+1}} \int_{\xi_{1}, l}^{\xi_{1}: l+1} \partial_{3} \widetilde{G}_{r s, 2}\left(0,0, x_{3}^{j}, \xi_{1}, 0, \xi_{3} ; \omega\right) \mathrm{d} \xi_{1} \mathrm{~d} \xi_{3} .
\end{aligned}
$$

where $\xi_{1}^{i, l}=\xi_{1}^{\prime l}-\left(\xi_{1}^{\prime}\right)_{O}$.

Appendix D Expressions for $I_{i}^{(\alpha-1)}, I_{i, j}^{(\alpha-1)}$ and $I_{5,3}^{(\alpha-1)}(\alpha=1,2, i=1,2,3,4$ and $j=1,2$, 3)

$$
\begin{aligned}
& \left\{\begin{array}{l}
I_{1}^{(0)} \\
I_{3}^{(0)}
\end{array}\right\}=\frac{1}{2} \int_{0}^{+\infty} A_{S}^{(1)}\left(x_{3}, \xi_{3}, k, \omega\right)\left[J_{0}(k r) \mp J_{2}(k r)\right] \frac{1}{k} \mathrm{~d} k, \\
& \left\{\begin{array}{l}
I_{2}^{(0)} \\
I_{4}^{(0)}
\end{array}\right\}=\frac{1}{2} \int_{0}^{+\infty} A_{T}^{(1)}\left(x_{3}, \xi_{3}, k, \omega\right)\left[J_{0}(k r) \pm J_{2}(k r)\right] \frac{1}{k} \mathrm{~d} k, \\
& I_{5}^{(0)}=\int_{0}^{+\infty} A_{R}^{(1)}\left(x_{3}, \xi_{3}, k, \omega\right) J_{1}(k r) \frac{1}{k} \mathrm{~d} k, \\
& \left\{\begin{array}{c}
I_{1}^{(1)} \\
I_{4}^{(1)}
\end{array}\right\}=\int_{0}^{+\infty}\left\{\begin{array}{c}
A_{S}^{(1)} \\
A_{T}^{(1)}
\end{array}\right\}\left(x_{3}, \xi_{3}, k, \omega\right)\left[\frac{1}{k r} J_{2}(k r)-J_{1}(k r)\right] \mathrm{d} k, \\
& \left\{\begin{array}{c}
I_{2}^{(1)} \\
I_{3}^{(1)}
\end{array}\right\}=-\int_{0}^{+\infty} \frac{1}{k r}\left\{\begin{array}{c}
A_{T}^{(1)} \\
A_{S}^{(1)}
\end{array}\right\}\left(x_{3}, \xi_{3}, k, \omega\right) J_{2}(k r) \mathrm{d} k, \\
& I_{5}^{(1)}=\int_{0}^{+\infty} A_{R}^{(1)}\left(x_{3}, \xi_{3}, k, \omega\right) J_{1}^{\prime}(k r) \mathrm{d} k, \\
& I_{1,2}^{(0)}=\frac{\gamma_{2}}{4} \int_{0}^{+\infty} A_{S}^{(1)}\left(x_{3}, \xi_{3}, k, \omega\right)\left[3 J_{1}(k r)-J_{3}(k r)\right] \mathrm{d} k, \\
& I_{2,2}^{(0)}=\frac{\gamma_{2}}{4} \int_{0}^{+\infty} A_{T}^{(1)}\left(x_{3}, \xi_{3}, k, \omega\right)\left[J_{1}(k r)+J_{3}(k r)\right] \mathrm{d} k, \\
& I_{3,2}^{(0)}=\frac{\gamma_{2}}{4} \int_{0}^{+\infty} A_{S}^{(1)}\left(x_{3}, \xi_{3}, k, \omega\right)\left[J_{1}(k r)+J_{3}(k r)\right] \mathrm{d} k, \\
& I_{4,2}^{(0)}=\frac{\gamma_{2}}{4} \int_{0}^{+\infty} A_{T}^{(1)}\left(x_{3}, \xi_{3}, k, \omega\right)\left[3 J_{1}(k r)-J_{3}(k r)\right] \mathrm{d} k, \\
& I_{1,2}^{(1)}=\frac{\gamma_{2}}{8} \int_{0}^{+\infty} A_{S}^{(1)}\left(x_{3}, \xi_{3}, k, \omega\right)\left[3 J_{0}(k r)-4 J_{2}(k r)+J_{4}(k r)\right] k \mathrm{~d} k,
\end{aligned}
$$




$$
\begin{gathered}
I_{2,2}^{(1)}=\frac{\gamma_{2}}{8} \int_{0}^{+\infty} A_{T}^{(1)}\left(x_{3}, \xi_{3}, k, \omega\right)\left[J_{0}(k r)-J_{4}(k r)\right] k \mathrm{~d} k, \\
I_{3,2}^{(1)}=\frac{\gamma_{2}}{8} \int_{0}^{+\infty} A_{S}^{(1)}\left(x_{3}, \xi_{3}, k, \omega\right)\left[J_{0}(k r)-J_{4}(k r)\right] k \mathrm{~d} k, \\
I_{4,2}^{(1)}=\frac{\gamma_{2}}{8} \int_{0}^{+\infty} A_{T}^{(1)}\left(x_{3}, \xi_{3}, k, \omega\right)\left[3 J_{0}(k r)-4 J_{2}(k r)+J_{4}(k r)\right] k \mathrm{~d} k, \\
\left\{\begin{array}{l}
I_{1,3}^{(0)} \\
I_{3,3}^{(0)}
\end{array}\right\}=\frac{1}{2} \int_{0}^{+\infty} A_{S}^{(2)}\left(x_{3}, \xi_{3}, k, \omega\right)\left[J_{0}(k r) \mp J_{2}(k r)\right] \mathrm{d} k, \\
\left\{\begin{array}{l}
I_{2,3}^{(0)} \\
I_{4,3}^{(0)}
\end{array}\right\}=\frac{1}{2} \int_{0}^{+\infty} A_{T}^{(2)}\left(x_{3}, \xi_{3}, k, \omega\right)\left[J_{0}(k r) \pm J_{2}(k r)\right] \mathrm{d} k, \\
\left\{\begin{array}{l}
I_{1,3}^{(1)} \\
I_{4,3}^{(1)}
\end{array}\right\}=\int_{0}^{+\infty}\left\{\begin{array}{l}
A_{S}^{(2)} \\
A_{T}^{(2)}
\end{array}\right\}\left(x_{3}, \xi_{3}, k, \omega\right)\left[\frac{1}{k r} J_{2}(k r)-J_{1}(k r)\right] k \mathrm{~d} k, \\
\left\{\begin{array}{l}
I_{2,3}^{(1)} \\
I_{3,3}^{(1)}
\end{array}\right\}=-\int_{0}^{+\infty} \frac{1}{k r}\left\{\begin{array}{l}
(0) \\
A_{T}^{(2)} \\
A_{S}^{(2)}
\end{array}\right\}\left(x_{3}, \xi_{3}, k, \omega\right) J_{2}(k r) k \mathrm{~d} k, \\
A_{R}^{(2)}\left(x_{3}, \xi_{3}, k, \omega\right) J_{1}^{\prime}(k r) k \mathrm{~d} k, \\
A_{R}^{(2)}\left(x_{3}, \xi_{3}, k, \omega\right) J_{1}(k r) \mathrm{d} k,
\end{gathered}
$$

where

$$
\begin{aligned}
& A_{S}^{(1)}\left(x_{3}, \xi_{3}, k, \omega\right)=-\frac{k^{2} \beta^{2}}{\omega^{2}}\left\{\frac{\bar{R}(k)}{R(k)}\left(\frac{k^{2}}{\gamma} e_{P P}+v e_{s s}\right)-\frac{4 k^{2} v \chi}{R(k)}\left(e_{S P}+e_{P S}\right)\right\}, \\
& A_{R}^{(1)}\left(x_{3}, \xi_{3}, k, \omega\right)=-\frac{k^{3} \beta^{2}}{\omega^{2}}\left\{\frac{\bar{R}(k)}{R(k)}\left(e_{P P}+e_{s S}\right)-\frac{4 \chi}{R(k)}\left(v \gamma e_{S P}+k^{2} e_{P S}\right)\right\} \\
& A_{S}^{(2)}\left(x_{3}, \xi_{3}, k, \omega\right)=\frac{k \beta^{2}}{\omega^{2}}\left\{\frac{\bar{R}(k)}{R(k)}\left(k^{2} e_{P P}+v^{2} e_{s s}\right)-\frac{4 k^{2} v \chi}{R(k)}\left(x_{S P}+v e_{P S}\right)\right\} \\
& A_{R}^{(2)}\left(x_{3}, \xi_{3}, k, \omega\right)=\frac{k^{2} \beta^{2}}{\omega^{2}}\left\{\frac{\bar{R}(k)}{R(k)}\left(\not e_{P P}+v e_{s S}\right)-\frac{4 v \chi}{R(k)}\left(\gamma^{2} e_{S P}+k^{2} e_{P S}\right)\right\} \\
& A_{T}^{(2)}\left(x_{3}, \xi_{3}, k, \omega\right)=-k e_{S S},
\end{aligned}
$$

with $e_{P P}=e^{-\gamma\left(x_{3}+\xi_{3}\right)}, e_{S S}=e^{-v\left(x_{3}+\xi_{3}\right)}, e_{S P}=e^{-\left(\varkappa_{3}+v \xi_{3}\right)}$, and $e_{P S}=e^{-\left(\gamma \xi_{3}+\nu x_{3}\right)} . J_{n}(x)$ is $n^{\text {th }}$-order Bessel function, $\gamma=$ $\sqrt{k^{2}-(\omega / \alpha)^{2}}(\operatorname{Re}(\gamma)>0), \quad v=\sqrt{k^{2}-(\omega / \beta)^{2}}(\operatorname{Re}(v)>0), \quad \chi=2 k^{2}-(\omega / \beta)^{2}, R(k)=\chi^{2}-4 k^{2} v \gamma \quad$ and $\quad \bar{R}(k)=$ $\chi^{2}+4 k^{2} v \gamma . \alpha$ and $\beta$ are P- and S-wave velocity, respectively. 